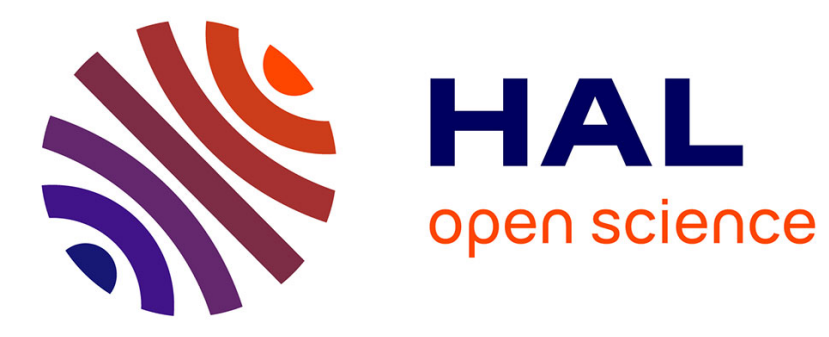

\title{
Design of a Magnetic Vibration Absorber with Tunable Stiffnesses
}

\author{
Simon Benacchio, Arnaud Malher, Jean Boisson, Cyril Touzé
}

\section{To cite this version:}

Simon Benacchio, Arnaud Malher, Jean Boisson, Cyril Touzé. Design of a Magnetic Vibration Absorber with Tunable Stiffnesses. Nonlinear Dynamics, 2016, 85 (2), pp.893-911. 10.1007/s11071-0162731-3 . hal-01286056

\section{HAL Id: hal-01286056 \\ https://hal-ensta-paris.archives-ouvertes.fr/hal-01286056}

Submitted on 10 Mar 2016

HAL is a multi-disciplinary open access archive for the deposit and dissemination of scientific research documents, whether they are published or not. The documents may come from teaching and research institutions in France or abroad, or from public or private research centers.
L'archive ouverte pluridisciplinaire HAL, est destinée au dépôt et à la diffusion de documents scientifiques de niveau recherche, publiés ou non, émanant des établissements d'enseignement et de recherche français ou étrangers, des laboratoires publics ou privés. 


\title{
Design of a Magnetic Vibration Absorber with Tunable Stiffnesses
}

\author{
S. Benacchio · A. Malher · J. Boisson • C. Touzé
}

Received: date / Accepted: date

\begin{abstract}
The design and characterisation of a magnetic vibration absorber (MVA), completely relying on magnetic forces, is addressed. A distinctive feature of the absorber is the ability of tuning the linear stiffness together with the nonlinear cubic and quintic stiffnesses by means of repulsive magnets located in the axis of the main vibrating magnetic mass, together with a set of corrective magnets located off the main axis. The tuning methodology is passive and relies only on three geometrical parameters. Consequently the MVA can be adjusted to design either a nonlinear tuned vibration absorber (NLTVA), a nonlinear energy sink (NES), or a bi-stable absorber with negative linear stiffness. The expressions of the stiffnesses are given from a multipole expansion of the magnetic fields of repulsive and corrective magnets. A complete static and dynamic characterisation is performed, showing the robustness of the modelling together with the ability of the MVA to work properly in different vibratory regimes, thus making it a suitable candidate for passive vibration mitigation in a wide variety of contexts.
\end{abstract}

Keywords Magnetic vibration absorber · tunable linear and nonlinear stiffnesses

\section{Introduction}

The vibration mitigation of mechanical structures is a long standing problem which receives a considerable and continuous interest due to its importance in engineering applications. The most famous vibration absorber is the linear Tuned-Mass Damper (TMD), originally proposed by Frahm [10] and theoretically studied by den Hartog in 1934 [20]. Whereas the original work by Ormondroyd and den Hartog considered the primary structure (PS) as a linear undamped oscillator [33], further research extended their results to a damped oscillator as PS, in order to derive the optimal parameter values for a given set of optimisation criteria $[40,3,48]$. The extension of these results to flexible structures having an infinite number of modes is also a topic for current research, see e.g. [24].

The drawbacks of the TMD as a vibration absorber are well known and documented. The main associated problem is related to the narrow bandwidth of optimal control, asking for an accurate tuning which must continue over times. In the last decades, a number of investigations have been conducted in order to overcome these limitations, one of the main ideas being to use a nonlinearity in order to improve the vibration absorber design. The concept of a Nonlinear Energy Sink (NES) relies on a vanishing linear stiffness, giving rise to an essentially nonlinear restoring force. Having no natural frequency, the NES can thus adapt itself to the frequency of the PS $[11,41,44]$. A targeted energy transfer (TET) can then occur in an irreversible fashion [42,43]. The concept has been demonstrated theoretically and experimentally in a series of papers $[31,27,26,14,25,32,12]$.

As also remarked by a number of investigators, the frequency range over which a linear passive vibration isolator is effective, may be limited by the mount stiffness required to support a static load. Hence, the idea of selecting the eigenfrequency of the vibration isolator as small as possible lead to the concept of "quasi-zero stiffness" (QZS) absorber, which has been studied both theoretically and experimentally $[6,23,5,46]$.

Another idea which has emerged recently is to use the nonlinearity of the absorber in order to control the nonlinearity of the primary structure. As remarked in [45], a TMD is less effective as soon as the PS is nonlinear and exhibit typical features of nonlinear dynamics. In this context, an extension of den Hartog's equal peak

S. Benacchio · A. Malher · J. Boisson · C. Touzé

IMSIA (Institute of Mechanical Sciences and Industrial Applications),

ENSTA ParisTech-CNRS-EDF-CEA,

Université Paris-Saclay,

828 Boulevard des maréchaux, 91762 Palaiseau Cedex, France 
method has been proposed in [17], leading to the definition of a nonlinear tuned vibration absorber (NLTVA), which can be used e.g. for suppressing the limit cycle oscillations in a Duffing-Van der Pol system [18].

A recognised drawback of the NES is that a sufficient energy level is required for the targeted energy transfer to occur $[42,43]$. Recent studies have considered the case of a negative linear stiffness, leading to a bi-stable vibration absorber device. For example, it has been shown in [29,37] that such a vibration absorber may be more efficient than an NES as the energy barrier for activation is smaller in the bi-stable case.

Numerous experimental devices have been proposed including for example thin rods with no pretension for an NES [31], or vibro-impact oscillator [2,13]. For the absorber with quasi-zero stiffness, the case of Euler buckled beams have been proposed in [28]. Recent realisations of the NLTVA have been built with 3D printed beams having a particular cross-section in order to correctly tune linear and nonlinear characteristics $[15,16]$. The vibration suppression of beams using eddy current dampers have been proposed in $[38,4,9]$ for pendulum and normal motions respectively. A variety of nonlinear absorbers of more general forms have also been proposed including for example the case of centrifugal pendulum for torsional vibration mitigation [19].

In this contribution, a passive magnetic vibration absorber (MVA) is proposed with the ability of properly tuning its linear and nonlinear characteristics. Our goal is to propose a flexible device that can be used either as an NES, an NLTVA, or a bi-stable vibration absorber, all these tunings being realised passively with a simple change in the system's geometry. The device relies on the use of magnetic forces in order to be able to tune the stiffnesses. The idea of using magnetic forces for designing a vibration absorber can be traced back to the pioneering work of Yamakawa and Kojima $[47,22,21]$. In these articles, they use a simple arrangement with a mobile magnet at centre in the repulsive magnetic fields of fixed magnets. The device being vertical, the gravity force was also taken into account (the center magnet being levitating), in order to design a QZS absorber [36]. Other arrangements where the correction forces are provided by springs have also been studied in $[7,46]$. Moreover, the study of nonlinear magnetic systems goes over the vibration reduction field with energy harvesting devices, see for example [30]. Finally, Al-Shudeifat proposes an asymmetric arrangement of the initial design of Yamakawa and Kojima in order to realise an NES [1]. In general, we note that the realisation of vibration absorber devices are not flexible and are tuned for the purpose of realising a specific case, TMD, NES, NLTVA, or bi-stable.

The paper is organised as follows. In the first section we introduce the geometry of the device. The basic idea is the same as those already used since Yamakawa and Kojima, the originality being the use of a set of four fixed corrective magnets placed at an offset position of the main axis. A multipole expansion is used to model the magnetic forces, so that the linear, cubic and quintic nonlinear stiffnesses can be evaluated. Their variations with respect to geometric parameters are studied in order to show how one can select the desired absorber: NES, NLTVA or bi-stable. Static and dynamic tests are then experimentally conducted in order to obtain a complete characterisation of the MVA.

\section{The Magnetic Vibration Absorber (MVA)}

\subsection{Main Characteristics}

The proposed vibration absorber is shown in Fig. 1. It is composed of 7 permanent ring magnets located on

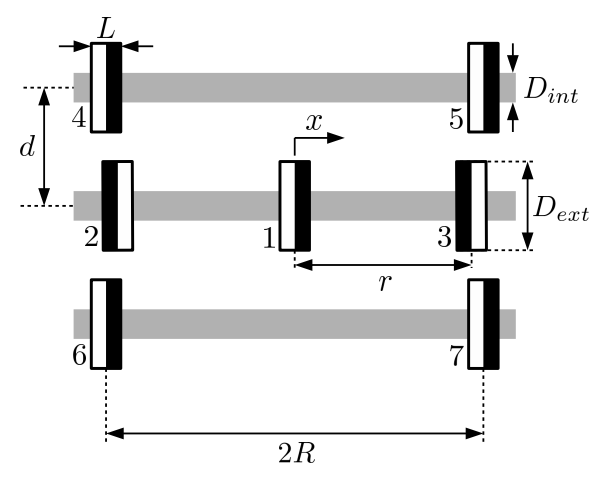

Fig. 1 Schematic representation of the magnetic vibration absorber (MVA).

plastic rods, shown in grey in Fig. 1. Their length, internal and external diameters are denoted $L, D_{\text {int }}$ and $D_{\text {ext }}$ respectively. The mass of the absorber oscillating around the central position $x=0$ is given by the magnet 1 . This mass can be easily tuned by adding a massive object at center as proposed e.g. in [2]. The main vibration axis is horizontal so that the gravity has no influence on the restoring force exerted on magnet 1 . The other 
magnets, respectively numbered $2,3,4,5,6$ and 7, are fixed on the rods. The black and white parts of each magnet indicates the direction of their axial magnetisation. Regarding the main magnet (1), magnets 2 and 3 have an opposite magnetisation. As they apply a repulsive force on the magnet 1, pushing it in its central position, they are named the repulsive magnets. The amplitude of the repulsive force can be tuned by modifying the geometric parameter $r$, or the magnetisation. Magnets 4, 5, 6 and 7, named the corrective magnets, have the same direction of magnetisation as magnet 1 and apply an attractive force on it. The amplitude of this force is adjustable by modifying the geometric parameters $R$ and $d$. Therefore, the total force applied on the magnet 1 is tuned by the independent modifications of the geometric parameters $r, R$ and $d$.

\subsection{Experimental Realisation}

Numerous experimental configurations can be built following the model of the proposed MVA, and a number of them have been tested in the laboratory in order to characterize the MVA. In this section we focus on a practical realization, convenient for a number of static and dynamical tests. A picture of this selected device is shown in Fig. 2. In particular, the same type of ring magnets has been retained for vibrating, repulsive and corrective magnets. The characteristics of these ring magnets are given in Table 1. Of course, different magnets

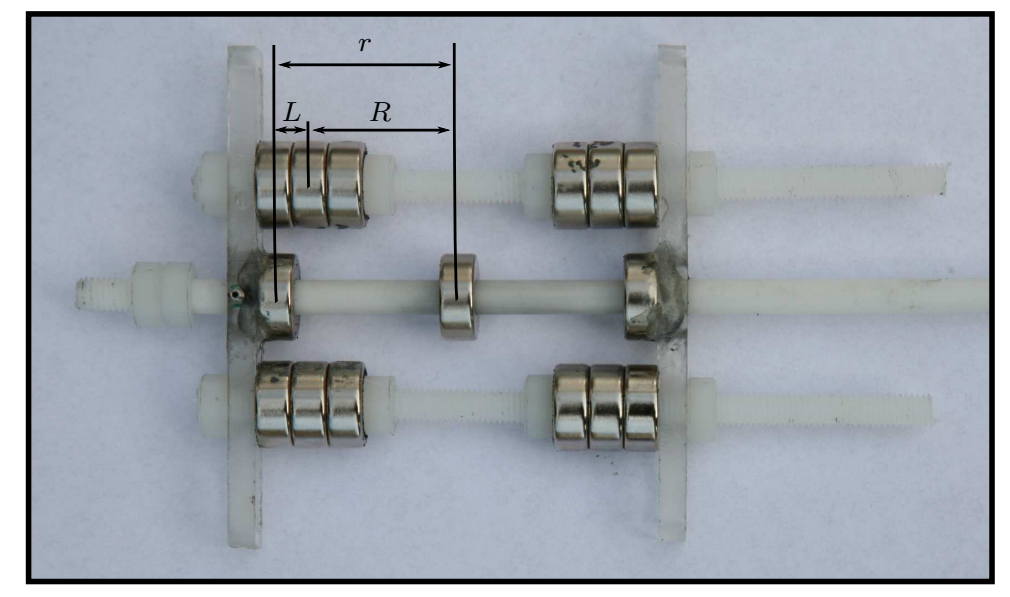

Fig. 2 A realization of the magnetic vibration absorber with the definition of the additional length $L$.

can be used in the device for tuning the desired properties. To get a corrective force that is large enough in

\begin{tabular}{cccccc} 
Length $(L)$ & Internal Diameter $\left(D_{\text {int }}\right)$ & External Diameter $\left(D_{\text {ext }}\right)$ & Weight $\left(M_{\text {mag }}\right)$ & Type & Grade \\
\hline $5 \mathrm{~mm}$ & $10 \mathrm{~mm}$ & $20 \mathrm{~mm}$ & $10 \mathrm{~g}$ & $\mathrm{NdFeB}$ & $\mathrm{N} 42$
\end{tabular}

Table 1 Characteristics of the magnets.

comparison to the repulsive force, the corrective magnets are in fact composed of 3 rings stuck together, in order to increase their total magnetic moments. So as to fit with the schematic representation of Fig. 1, the length $2 R$ corresponds to the distance between the two magnetic moments of the corrective magnets located on the same axis. The magnetic moment is assumed to be located at the centre of the 3 stuck magnets. Hence, $R$ represents the distance between the middle of the three corrective magnets and the central position, as shown in Fig. 2. Consequently, in the experimental realisation, the length $R$ is set equal to $r-L$, with $L$ the thickness of one magnet, see Fig. 2. As this experimental device has been mostly used in the remainder of the study, we will generally select $R$ as $R=r-L$.

\subsection{Theoretical Expression of the Stiffnesses}

Let us now derive the expression of the force applied on magnet 1. The mutual energy of two similar permanent magnets with a magnetic moment $\boldsymbol{m}$, a volume $V$ and creating a magnetic field $\boldsymbol{B}$ is given by

$$
E_{p}=-\int_{V} \boldsymbol{M} \cdot \boldsymbol{B} d V
$$


where $\boldsymbol{M}=\boldsymbol{m} / V$ is the corresponding uniform magnetisation. The force created by the magnetic field $\boldsymbol{B}(\boldsymbol{p})$ on the vibrating magnet is given by

$$
\boldsymbol{F}(\boldsymbol{p})=\nabla(\boldsymbol{m} \cdot \boldsymbol{B}(\boldsymbol{p}))
$$

where $\boldsymbol{p}$ is the coordinates of the magnet 1 relative to the fixed magnet as shown in Fig. 3. In this study,
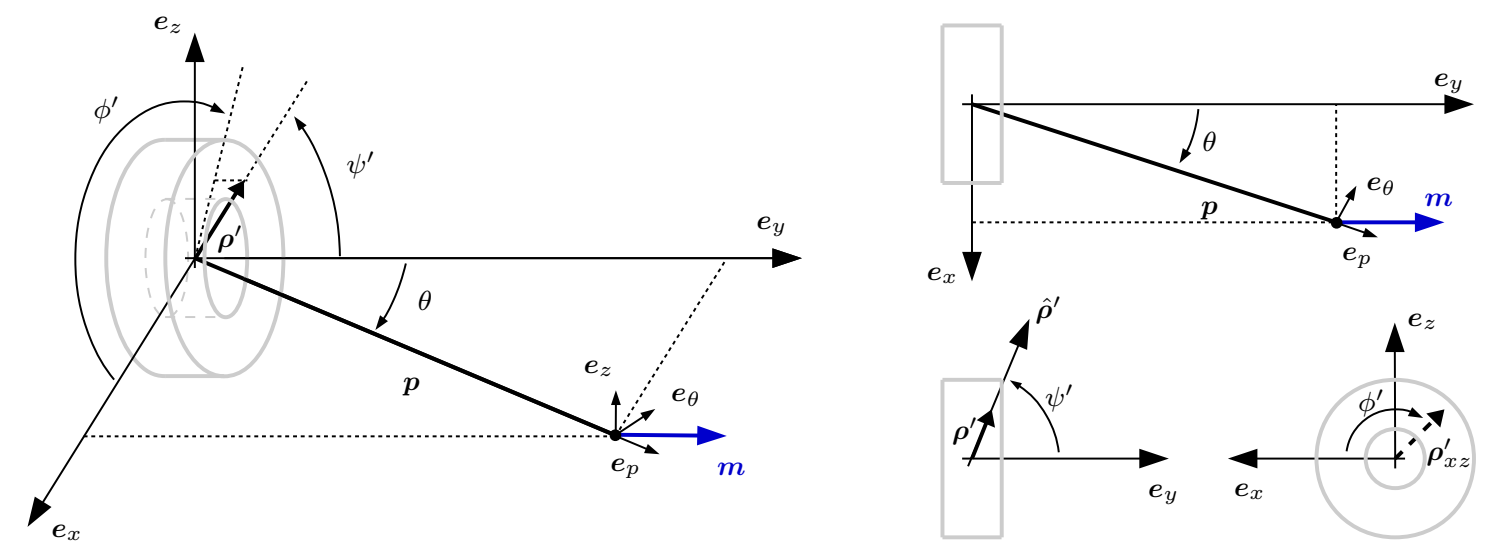

Fig. 3 Geometrical representation for computing the magnetic force applied on the moment $\boldsymbol{m}$, located at $\boldsymbol{p}$ and created by the ring magnet of external diameter $D_{\text {ext }}$, internal diameter $D_{i n t}$ and length $L$. Angles are defined such as $\phi^{\prime}=\left(\widehat{\boldsymbol{e}_{x}, \boldsymbol{\rho}_{x z}^{\prime}}\right)$ with $\boldsymbol{\rho}_{x z}^{\prime}$ the orthogonal projection of $\boldsymbol{\rho}^{\prime}$ in the plane $\left(\boldsymbol{e}_{x}, \boldsymbol{e}_{z}\right), \psi^{\prime}=\left(\widehat{\boldsymbol{e}_{y}, \boldsymbol{\rho}^{\prime}}\right)$ and $\theta=\left(\widehat{\boldsymbol{e}_{y}, \boldsymbol{p}}\right)$.

a multipole expansion is used to give the expression of the magnetic field in order to improve the dipole approximation which could give inaccurate results for small distance between magnets.

\subsubsection{Multipole Expansion}

The multipole expansion is briefly presented here for cylindrical permanent magnets axially magnetised. The calculation is first presented for a cylindrical magnet without hole. The hole is taken into account at the end of the calculation, by adding the magnetic field created by another cylindrical permanent magnet with opposite magnetisation, and a diameter equal to that of the hole. The interested reader is referred to [35] for a more detailed presentation of magnetic forces and multipole expansion. The used approach is known to be relevant if the distance between the magnets is larger than their characteristic lengths (usually the radius of a sphere containing the magnet). Fig. 3 gives the geometric parameters used for the computation when the fixed magnet is located at the origin of the basis $\left(\boldsymbol{e}_{x}, \boldsymbol{e}_{y}, \boldsymbol{e}_{z}\right)$ and the magnet 1 at the origin of the basis $\left(\boldsymbol{e}_{p}, \boldsymbol{e}_{\theta}, \boldsymbol{e}_{z}\right)$. All primed variables are defined relative to the fixed magnet for integration. The magnetic field reads

$$
\boldsymbol{B}(\boldsymbol{p})=\frac{\mu_{0} m}{4 \pi V} \sum_{n \text { odd }} \frac{(L / 2)^{n+2}}{p^{n+2}}\left((n+1) D_{n} \boldsymbol{e}_{p}-\frac{d D_{n}}{d \theta} \boldsymbol{e}_{\theta}\right)=\sum_{n \text { odd }} \boldsymbol{B}_{n}(\boldsymbol{p})
$$

where $\mu_{0}$ is the void permeability, $p$ and $m$ are respectively the magnitudes of the vector $\boldsymbol{p}$ and of the magnetic moment $\boldsymbol{m}$ of the fixed magnet. Using the substitution $\gamma=\cos \left(\psi^{\prime}\right)$, the term $\left((n+1) D_{n} \boldsymbol{e}_{p}-\frac{d D_{n}}{d \theta} \boldsymbol{e}_{\theta}\right)$ takes into account the shape of the fixed magnet with

$$
D_{n}(\boldsymbol{p}, \beta)= \begin{cases}2 \int_{\frac{1}{\sqrt{1+\beta^{2}}}}^{1} \int_{0}^{2 \pi} \frac{P_{n}\left(\hat{\boldsymbol{p}} \cdot \hat{\boldsymbol{\rho}}^{\prime}\right)}{\gamma^{n+3}} d \phi^{\prime} d \gamma, & n \text { odd } \\ 0, & n \text { even }\end{cases}
$$

where $\beta=D_{\text {ext }} / L$ if the magnet is a fully solid cylinder and $P_{n}()$ the Legendre polynomials. Using the notations of Fig. 3, $\left(\hat{\boldsymbol{p}} . \hat{\boldsymbol{\rho}}^{\prime}\right)=\sqrt{1-\gamma^{2}} \sin \theta \cos \phi^{\prime}$, with $\hat{\boldsymbol{p}}=\boldsymbol{p} /\|\boldsymbol{p}\|$ and $\hat{\boldsymbol{\rho}}^{\prime}=\boldsymbol{\rho}^{\prime} /\left\|\boldsymbol{\rho}^{\prime}\right\|$ the unit vector of the integration point in the fixed magnet. 
Finally, rewriting $m \cos (\theta) \boldsymbol{e}_{p}=\left(\hat{\boldsymbol{p}} \hat{\boldsymbol{p}}^{T}-\boldsymbol{I} \boldsymbol{d}\right) \boldsymbol{m}$ and $m \sin (\theta) \boldsymbol{e}_{\theta}=\hat{\boldsymbol{p}} \hat{\boldsymbol{p}}^{T} \boldsymbol{m}$, the first terms of the magnetic field read

$$
\begin{aligned}
& \boldsymbol{B}_{1}(\boldsymbol{p})=\frac{\mu_{0}}{4 \pi} \frac{1}{p^{3}}\left[3 \hat{\boldsymbol{p}} \hat{\boldsymbol{p}}^{T}-\boldsymbol{I d}\right] \boldsymbol{m}, \\
& \boldsymbol{B}_{3}(\boldsymbol{p})=\frac{\mu_{0}}{4 \pi} \frac{1}{p^{5}}\left(\frac{L}{2}\right)^{2}\left(\frac{4-3 \beta^{2}}{8}\right)\left[\left(35\left(\hat{\boldsymbol{m}}^{T} \hat{\boldsymbol{p}}\right)^{2}-15\right) \hat{\boldsymbol{p}} \hat{\boldsymbol{p}}^{T}-\left(15\left(\hat{\boldsymbol{m}}^{T} \hat{\boldsymbol{p}}\right)^{2}-3\right) \boldsymbol{I} \boldsymbol{d}\right] \boldsymbol{m}, \\
& \boldsymbol{B}_{5}(\boldsymbol{p})=\frac{\mu_{0}}{4 \pi} \frac{1}{p^{7}}\left(\frac{L}{2}\right)^{4}\left(\frac{15 \beta^{4}-60 \beta^{2}+24}{64}\right)\left[\left(231\left(\hat{\boldsymbol{m}}^{T} \hat{\boldsymbol{p}}\right)^{4}-210\left(\hat{\boldsymbol{m}}^{T} \hat{\boldsymbol{p}}\right)^{2}+35\right) \hat{\boldsymbol{p}} \hat{\boldsymbol{p}}^{T}\right. \\
& \left.-\left(105\left(\hat{\boldsymbol{m}}^{T} \hat{\boldsymbol{p}}\right)^{4}-70\left(\hat{\boldsymbol{m}}^{T} \hat{\boldsymbol{p}}\right)^{2}+5\right) \boldsymbol{I} \boldsymbol{d}\right] \boldsymbol{m}, \\
& \boldsymbol{B}_{7}(\boldsymbol{p})=\frac{\mu_{0}}{4 \pi} \frac{1}{p^{9}}\left(\frac{L}{2}\right)^{6}\left(\frac{64-336 \beta^{2}+280 \beta^{4}-35 \beta^{6}}{1024}\right) \\
& {\left[\left(6435\left(\hat{\boldsymbol{m}}^{T} \hat{\boldsymbol{p}}\right)^{6}-9009\left(\hat{\boldsymbol{m}}^{T} \hat{\boldsymbol{p}}\right)^{4}+3465\left(\hat{\boldsymbol{m}}^{T} \hat{\boldsymbol{p}}\right)^{2}+315\right) \hat{\boldsymbol{p}} \hat{\boldsymbol{p}}^{T}\right.} \\
& \left.-\left(3003\left(\hat{\boldsymbol{m}}^{T} \hat{\boldsymbol{p}}\right)^{6}-3465\left(\hat{\boldsymbol{m}}^{T} \hat{\boldsymbol{p}}\right)^{4}+945\left(\hat{\boldsymbol{m}}^{T} \hat{\boldsymbol{p}}\right)^{2}-35\right) \boldsymbol{I} \boldsymbol{d}\right] \boldsymbol{m},
\end{aligned}
$$

where $\boldsymbol{I} \boldsymbol{d}$ is the unit matrix and ${ }^{T}$ stands for the transpose. As shown in section 3.2, the first four terms (dipole, quadrupole, hexapole and octupole) of the magnetic field gives an accurate model for the MVA.

In order to take into account the shape of the magnet, the ring is defined by the addition of two cylinders of equal length $L$ [35]. The magnetic field of the largest is computed as previously using the diameter $D_{\text {ext }}$ and a positive magnetisation $\boldsymbol{M}$, whereas the magnetic field of the hole is computed using the diameter $D_{\text {int }}$ and a negative magnetisation $-\boldsymbol{M}$. The latter is simply computed replacing $D_{\text {ext }}$ by $D_{\text {int }}$ and $\boldsymbol{m}$ by $-\boldsymbol{m}$ in Eq. (5).

\subsubsection{Total Stiffness Force}

The total force applied on magnet 1 due to the repulsive and corrective magnets is computed using Eq. (2) for magnets 2 to 7 and finally reads

$$
\boldsymbol{F}_{\mathrm{Tot}}=\sum_{i=2}^{7} \boldsymbol{F}\left(\boldsymbol{p}_{\boldsymbol{i}}\right)
$$

where $\boldsymbol{p}_{\boldsymbol{i}}$ is the vector between the centre of the magnets $i$ and 1 .

For $i=(2,3)$ one has

$$
\left|\boldsymbol{p}_{\boldsymbol{i}}\right|=(r+x)
$$

while for $i=(4,5,6,7)$,

$$
\left|\boldsymbol{p}_{\boldsymbol{i}}\right|=\left((R+x)^{2}+d^{2}\right)^{1 / 2},
$$

where $x$ is the displacement of the magnet 1 around its central position. According to Eq. (7), the repulsive force is tuned using the length $r$ while Eq. (8) shows that the lengths $R$ and $d$ tune the corrective force. Truncating the Taylor series around $x=0$ at the fifth order, the total force applied on the magnet 1 can be written as

$$
\begin{aligned}
\boldsymbol{F}_{\mathrm{Tot}}(x) & \approx-\left(K_{1}^{r e p}(r)-K_{1}^{c o r}(R, d)\right) x-\left(K_{3}^{r e p}(r)-K_{3}^{c o r}(R, d)\right) x^{3}-\left(K_{5}^{r e p}(r)-K_{5}^{c o r}(R, d)\right) x^{5}, \\
& \approx-K_{1} x-K_{3} x^{3}-K_{5} x^{5} .
\end{aligned}
$$

This magnetic force is factorised to give a linear, a cubic and a quintic stiffness terms. Modifying the geometric parameters $r, R$, and $d$, these three terms can be tuned to change the properties of the MVA. The detailed expressions of these coefficients are given in Appendix A.

\subsubsection{Stiffness Cartography}

The aim of this section is to show how the geometry of the device, parametrised by $r, R$ and $d$, can be used for tuning the stiffnesses of the MVA. Fig. 4 shows the evolution of the coefficients $K_{1}, K_{3}$ and $K_{5}$ as a function of $r$ and $d$. As explained in section 2.2, $R$ is selected as $R=r-L$ in all this section. The central row of Fig. 4, i.e. Figs. 4(d-f), corresponds to the experimental device shown in Fig. 2 with $R=r-L$. In order to explore numerically the possible stiffnesses of the MVA, the first row, i.e. Figs 4(a-c), shows a case where a larger value of $R$ has been selected. More specifically, denoting $r_{0}$ the minimal distance numerically computed $\left(r_{0}=20 \mathrm{~mm}\right.$ in Fig. 4), the length $R$ has been increased of $r_{0}$. This minimum distance is arbitrarily chosen according to the model limitations. Then, in Figs. 4(g-i), the distance $R$ has been decreased so as to observe the effect of a stronger correction brought by the corrective magnets. In this case, $R$ has been decreased of $r_{0} / 2$.

The stiffness cartography has been computed from the model presented in the previous section, with as input parameters two different magnetic moments, $m_{r}$ and $m_{c}$, respectively for the repulsive and corrective magnets. 
The selection of the numerical values relies on an identification procedure for the MVA design shown in Fig. 2 and used as a reference case for the study of the stiffness variations. The parameter values are $m_{r}=0.89$ and $m_{c}=0.71$. Moreover a shift $\Delta d=3 \mathrm{~mm}$ is added to $d$ in order to better fit the model with the experimental realisation. The choice of this parameter is more deeply explained in section 3.2.
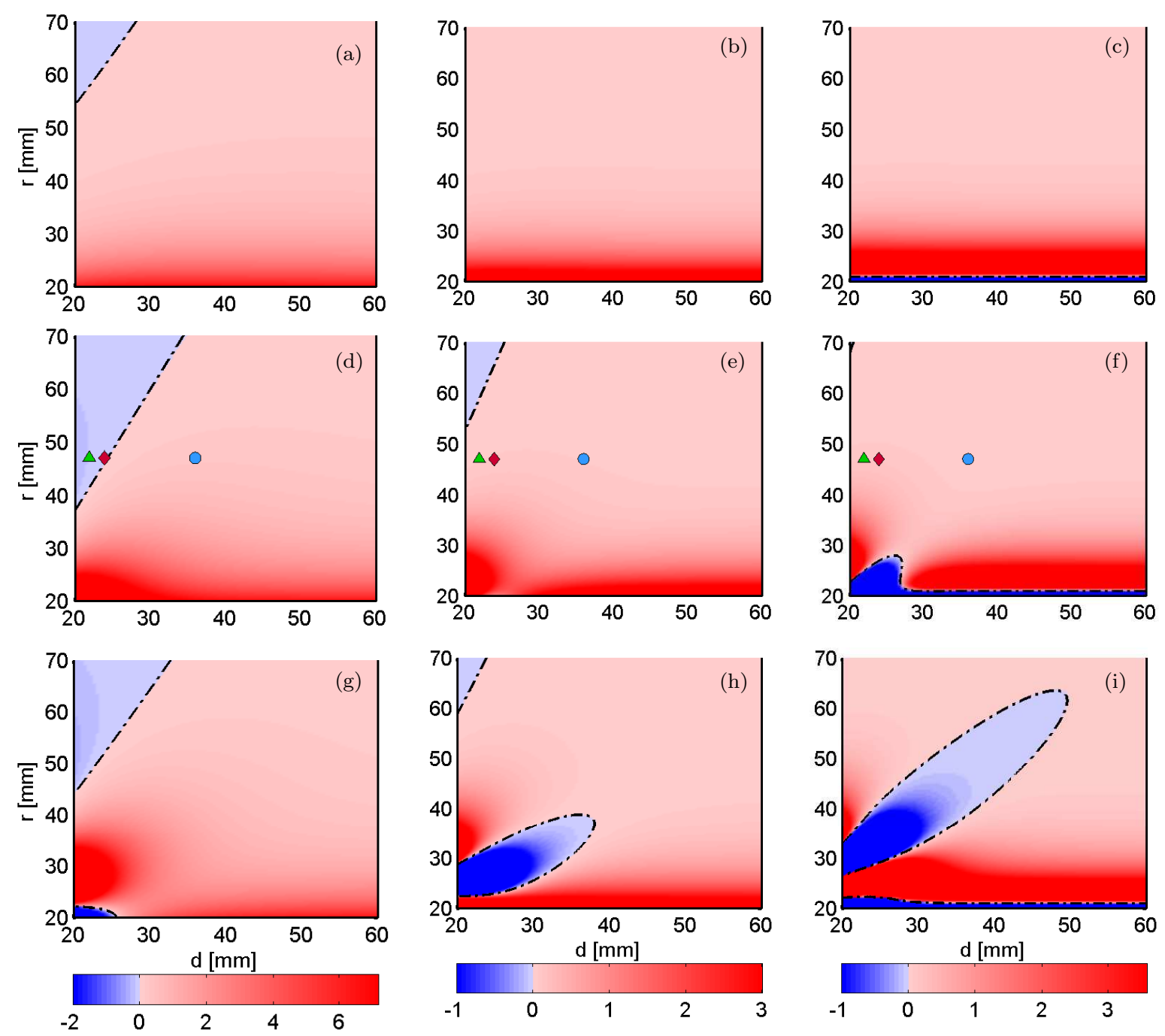

$K_{1} \cdot 10^{2}[\mathrm{~N} / \mathrm{m}]$

$K_{3} \cdot 10^{6}\left[\mathrm{~N} / \mathrm{m}^{3}\right]$

$K_{5} \cdot 10^{9}\left[\mathrm{~N} / \mathrm{m}^{5}\right]$

Fig. 4 Theoretical evolution of the linear (first column), cubic (second column) and quintic (third column) stiffness coefficients as functions of $r$ and $d$ for $R=r-L+20 \mathrm{~mm}(\mathrm{a}, \mathrm{b}, \mathrm{c}), R=r-L(\mathrm{~d}, \mathrm{e}, \mathrm{f})$ and $R=r-L-10 \mathrm{~mm}$ (g, h, i). Zeros are shown using black dashed lines. Configurations of Fig. 5 are denoted with the markers as: $\Delta, \diamond, \bullet$.

Figure 4 demonstrates that many stiffness configurations can be reached using the proposed MVA. Modifying the geometric parameters, all the stiffness coefficients can be either positive, equal to zero or negative. According to Figs. 4(a-c), for a large value of $R$, linear $\left(K_{1}\right)$ and quintic $\left(K_{5}\right)$ coefficients are positive in almost all geometric configurations while the cubic coefficient $K_{3}$ is always above zero. Largest values of the coefficients are found for small values of $r$. When the corrective magnets are far from the magnet 1 , the parameter $d$ has a small effect on stiffness coefficients. As shown in Figs. 4(d-f), when $R$ is roughly equal to $r$, more geometric configurations are able to produce negative coefficients. The parameter $d$ has now an important effect on the coefficients. For example, $K_{5}$ can be either positive or negative by modifying $d$ when $r=25 \mathrm{~mm}$, see Fig. 4(f). Finally, when $R$ is smaller than $r$ as in Fig. 4(g-i), the cartographies of the stiffness coefficients become more complex. Interestingly enough, one can notice that in this configuration, it is possible to have negative values for the two nonlinear coefficients and a positive value for $K_{1}$. This could be useful to tuned the MVA as an NLTVA for the damping of shells (or arches) vibrations. Indeed, for some values of the curvature, a shallow shell may exhibit a softening nonlinearity, see e.g. [34,39]. As the nonlinear term of an NLTVA should be tuned as the "mirror" of the nonlinearity of the primary structure to be damped $[45,17]$, then in this case one needs to design an NLTVA with a positive $K_{1}$ and a negative $K_{3}$. 
To sum up the results of Fig. 4, the linear coefficient is large for small values of $r$, or in other words when magnets are close together. Negative values of $K_{1}$ are given by large $r$. The extremum values of coefficients can be increased by modifying the magnetic properties of the device. For instance, a larger magnetisation may be used in order to increase the difference between minimum and maximum values of $K_{1}$. If one changes the magnet grade from N42 to N50, an increase of the linear stiffness of the order of about $10 \%$ can be awaited. Another option is to add more magnets to the absorber. As the magnetisation is proportional to the volume occupied by the magnetic material, a substantial increase in the forces can be obtained.

In the following sections, $R$ is equal to $r-L$ as in Figs. 4(d-f). In order to investigate the behaviour of the MVA, this paper focuses on the modification of the linear stiffness coefficient. In Fig. 4(d), the blue, red and green markers present geometric configurations where $K_{1}$ is respectively positive, equal to zero and negative. Hence in this three selected configurations, the MVA is tuned either as a vibration absorber (TVA, marker $\mathbf{\Delta}$ ), a nonlinear energy sink (NES, $\diamond$ ) or a bi-stable damper $(\bullet)$ respectively. In each case, the nonlinear coefficients are positive. The remainder of the study is focused on these three different realisations. Numerous other test configurations could have been investigated, by tuning the nonlinear characteristic of the MVA (in order to obtain hardening or softening nonlinearity), or by adjusting the magnetic parameters, or the mass of the MVA. However all these further case study are postponed to the next step of this research, where the MVA will be used in real conditions on a given structure.

\section{Static Behaviour}

The MVA is now experimentally studied. Static force measurements are reported, in order to investigate the behaviour of the absorber for the three cases indicated with the markers in Fig. 4. A thorough identification of the model parameters is then conducted.

\subsection{Static Force Measurements}

The proposed model of the tunable MVA is compared with static force measurements for the three geometric configurations indicated with the markers in Fig. 4 and described by $r$ and $d$ in Table 2. The magnet 1 is moved around its central position $x=0$ with a $1 \mathrm{~mm}$ step. The static force applied on the magnet is measured for each step and presented using blue crosses in Fig. 5. The inserts show the measurements around the central

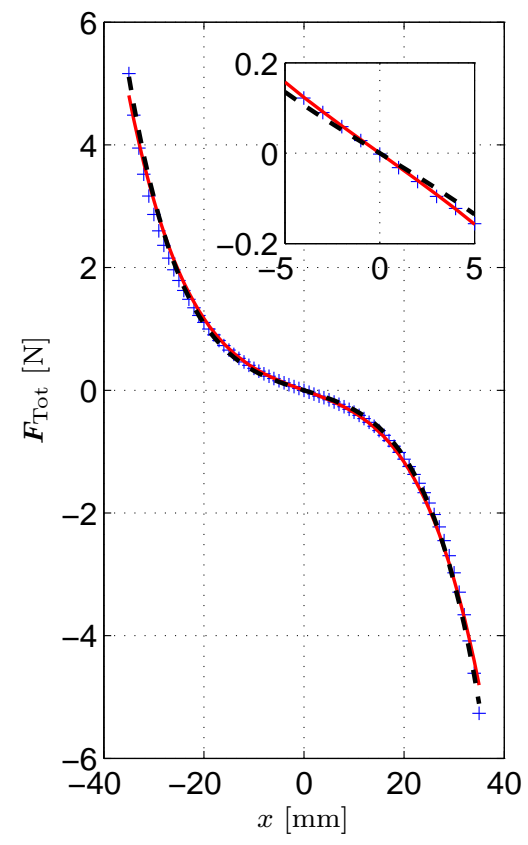

(a) $K_{1}>0(\Delta)$

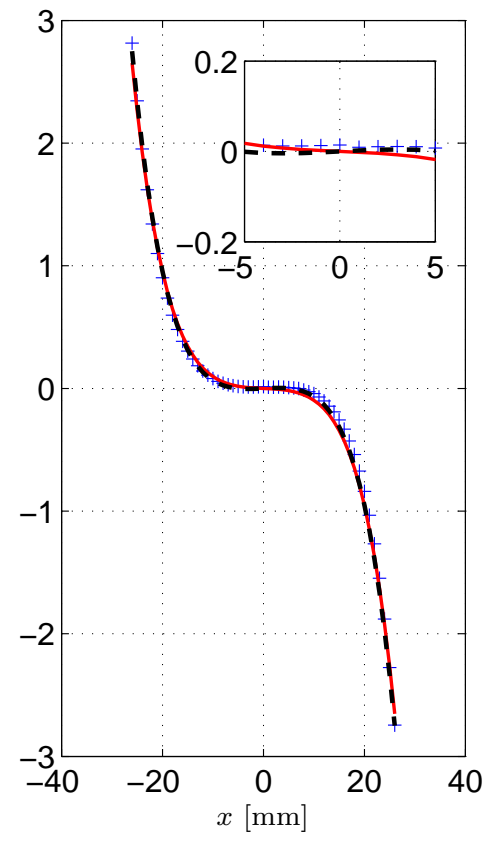

(b) $K_{1} \approx 0(\diamond)$

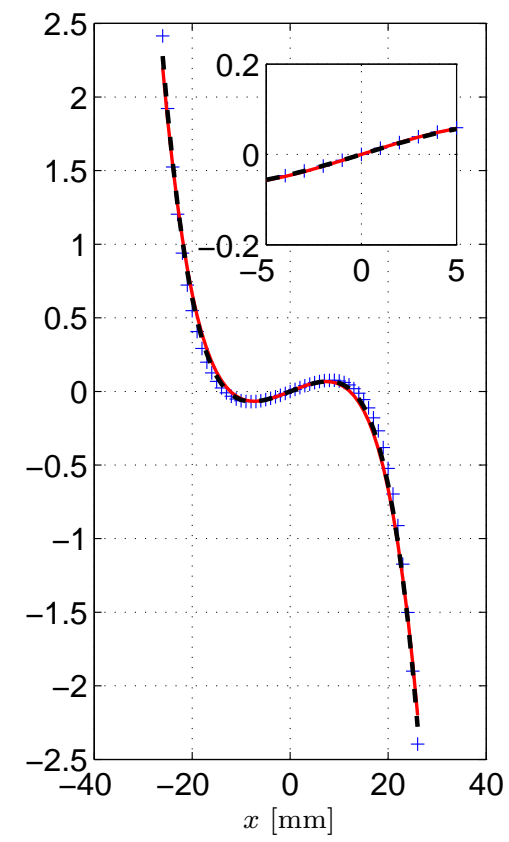

(c) $K_{1}<0(\bullet)$

Fig. 5 Static force applied on the magnet 1 as a function of its displacement. Measurement [+], experimental fit [ $\left.\_\right]$and theoretical fitted model [___- ] (see text). Colored markers refer to geometric configurations given in Table 2 and shown in Fig. 4. 
$K_{1}$ can be directly observed. Since the force is given by $\boldsymbol{F}_{\text {Tot }}(x) \approx-K_{1} x$ for small displacements, Figs. $5(\mathrm{a})$, $5(\mathrm{~b})$ and $5(\mathrm{c})$ show that the linear coefficient is respectively positive, quasi-equal to zero, and negative. These results are in agreement with the theoretical conclusions of Fig. 4(d) and demonstrate that the MVA can be experimentally tuned as a TVA, an NES or a bi-stable damper, which has a linear negative stiffness and thus possess two stable equilibria around the unstable central position.

\subsection{Identification of the Model Parameters}

In order to find the experimental values of the stiffness coefficients, the measurements are fitted using Eq. (10). For the three configurations the linear, cubic and quintic fitted terms, named $K_{1}^{E x p}, K_{3}^{E x p}$ and $K_{5}^{E x p}$, are given in Table 2. The plot of Eq. (10) using these values is presented in Fig. 5 and shows that this simple function is able to accurately estimate the behaviour of the MVA.

\begin{tabular}{cccc} 
Param. & Fig 5(a) & Fig 5(b) & Fig 5(c) \\
\hline \hline$r[\mathrm{~mm}]$ & 47 & 47 & 47 \\
$d[\mathrm{~mm}]$ & 36 & 24 & 22 \\
\hline \hline$K_{1}^{E x p}[\mathrm{~N} / \mathrm{m}]$ & 29.90 & 1.84 & -13.39 \\
$K_{1}[\mathrm{~N} / \mathrm{m}]$ & 25.59 & -2.11 & -12.77 \\
\hline$K_{3}^{E x p}\left[\mathrm{~N} / \mathrm{m}^{3}\right]$ & $0.6410^{5}$ & $0.6610^{5}$ & $0.7410^{5}$ \\
$K_{3}\left[\mathrm{~N} / \mathrm{m}^{3}\right]$ & $0.5910^{5}$ & $0.7310^{5}$ & $0.5710^{5}$ \\
\hline$K_{5}^{E x p}\left[\mathrm{~N} / \mathrm{m}^{5}\right]$ & $0.2010^{8}$ & $1.2110^{8}$ & $1.0410^{8}$ \\
$K_{5}\left[\mathrm{~N} / \mathrm{m}^{5}\right]$ & $0.3210^{8}$ & $1.2810^{8}$ & $1.3610^{8}$
\end{tabular}

Table 2 Experimental fitted and theoretical optimised stiffness coefficients.

Then, the experimental linear stiffness coefficients are used to optimise the model presented in section 2.3. In the expression of $K_{1}$ detailed in Appendix 1, three parameters are used for this optimisation. Since the theoretical value of the magnetic moment $m_{t h e o}$ depends on the approximate remanent magnetic field $B_{r}$ given by the manufacturers, the two first variable parameters are $m_{r}$ and $m_{c}$. Moreover, to take into account the errors of the length measurements, the third parameter is a shift $\Delta d$, added to the length $d$. This adjusting parameter is used as an overall free variable taking into account the measurement errors in geometry for each magnet which occur when the experimental realisation of the MVA is not perfectly symmetric. To optimise these parameters, the method of least squares using the experimental and the theoretical stiffness coefficients is applied for a large number of geometric configurations $N$. Thus, the cost function to minimise is

$$
\mathcal{F}_{\text {cost }}\left(m_{r}, m_{c}, \Delta d\right)=\sum_{i=1}^{N}\left(K_{1, i}^{E x p}-K_{1, i}\left(m_{r}, m_{c}, \Delta d\right)\right)^{2}=\sum_{i=1}^{N}\left(K_{1, i}^{E x p}-\left\{K_{1, i}^{r e p}\left(m_{r}, \Delta d\right)-K_{1, i}^{c o r}\left(m_{c}, \Delta d\right)\right\}\right)^{2}
$$

Fig. 6(a) shows the experimental linear coefficients for 50 different configurations used to find the optimal values of the variable parameters given in Table 3 .

\begin{tabular}{ccc}
$m_{r}$ & $m_{c}$ & $\Delta d$ \\
\hline 0.89 & 0.71 & $3 \mathrm{~mm}$
\end{tabular}

Table 3 Optimal values of the variable parameters.

In order to confirm these magnetic moment values found from the fitting procedure, magnetic field measurements along the axis of a ring magnet, as well as shifted from the same axis, have been conducted. These measurements clearly demonstrate that the optimized value of $m_{r}$ is in good agreement with the magnetic field measurements. Nevertheless, outside of the magnet axis, measurements show that the magnetic field deviates from the multipole expansion theory. This is the reason why an equivalent magnetic moment $m_{c}$, different from $m_{r}$, is introduced. Moreover it is in good agreement with the calculation of $K_{1}, K_{3}$ and $K_{5}$. For the three cases presented in section 3.1, the stiffness coefficients computed using the optimal model are given in Table 2 and shown with black dashed lines in Fig. 5. Since theoretical and experimental values are close, the optimal model is believed to give a reliable description of the MVA.

To conclude this section, a discussion on the accuracy of the multipole expansion as compared to the dipole approximation, is provided. For every configuration the theoretical stiffness coefficients are compared to the experimental results and presented in Fig. 6 with dashed and full lines respectively. Each point represents a geometric configuration corresponding to a pair $(r, d)$ linked together for the same $r$. The optimal model gives very accurate results for the linear stiffness which is always close to the experimental coefficient. When the 

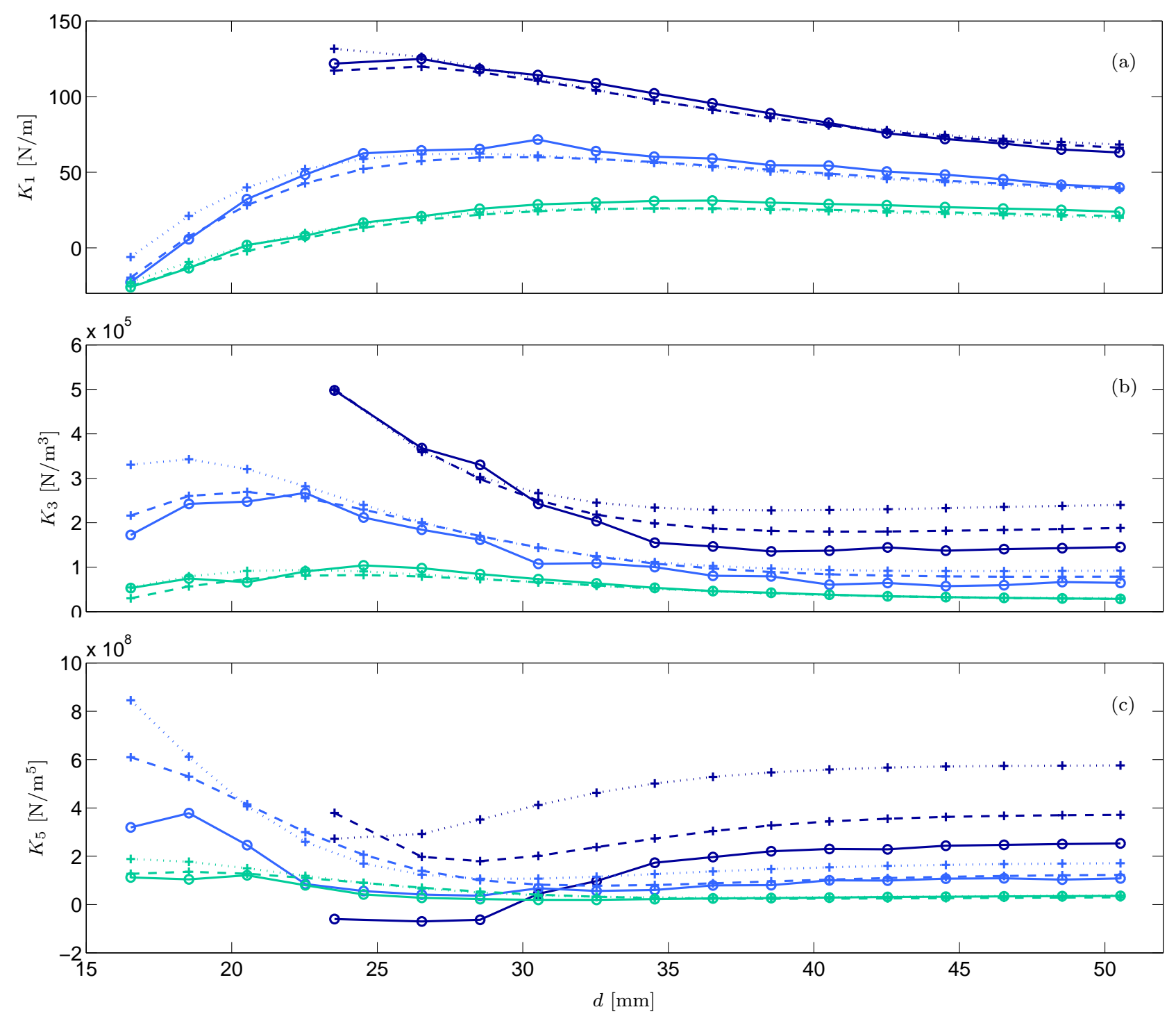

Fig. 6 Linear (a), cubic (b) and quintic (c) stiffness coefficients for $r=35 \mathrm{~mm}$ [ $\_$], $r=40 \mathrm{~mm}$ [ $]$ and $r=45 \mathrm{~mm}$ [ $]$ ]. Full lines are the results of the experimental fit, dotted lines are the results of a dipole approximation and dashed lines are the results of the multipole expansion.

distances $r$ and $d$ are large, the model gives also very accurate results for the nonlinear coefficients $K_{3}$ and $K_{5}$. The accuracy of the model decreases when $r$ and $d$ also decrease, see e.g. Fig. 6(b) and 6(c). For instance, $K_{5}$ is incorrectly predicted when $r=35 \mathrm{~mm}$, or $r=40 \mathrm{~mm}$ and $d<30 \mathrm{~mm}$, but the figure definitely shows that using a multipole expansion improves significantly the accuracy of the model for small values. Indeed, the dotted lines show the theoretical stiffness coefficients given by a dipole approximation, whereas the dashed line shows the result predicted with the multipole expansion. Note that when using only the dipole term, the optimisation procedure provides different (though close) values: $m_{r}=0.82, m_{c}=0.72$ and $\Delta d=3 \mathrm{~mm}$. Fig. 6(a) shows that the two models give similar results for the linear stiffness coefficients. However, the multipole expansion provides better results for the modelling of nonlinear coefficients as shown in Fig. 6(b) and 6(c), and even for small values of $r$ and $d$. For instance, when $r=35 \mathrm{~mm}$, the error on $K_{3}$ and $K_{5}$ is divided by 2 almost everywhere using the multipole expansion.

To conclude this section, the model proposed in section 2 gives accurate results for the modelling of the linear and cubic stiffness coefficients. For the quintic term the accuracy is ensured only for large values of $r$ and $d$. For almost every geometric configuration the multipole expansion is more accurate than the dipole approximation, this being especially true for nonlinear coefficients. 


\section{Dynamical Behaviour}

This section is devoted to the dynamical behaviour of the MVA, tuned either as an NLTVA, an NES or a bistable absorber. In order to investigate its general dynamics, experiments are compared to numerical simulations for the 3 geometric configurations. The MVA which has been used to study the NLTVA and the NES is the experimental realisation presented in section 2.2. Nevertheless, the linear stiffness of the NLTVA has been increased slightly in order to cope with experimental limitations in low-frequency range due to the use of a shaker. For practical reasons, the MVA selected to study the bi-stable absorber is built with smaller magnets. Thus, the characteristics of the new magnets are $L=6 \mathrm{~mm}, D_{\text {int }}=6 \mathrm{~mm}, D_{\text {ext }}=15 \mathrm{~mm}, m_{\text {mag }}=6.6 \mathrm{~g}$ and the results of the optimisation step give $m_{r}=0.67, m_{c}=0.54$ and $\Delta d=2 \mathrm{~mm}$.

4.1 Experimental set-up and model comparison

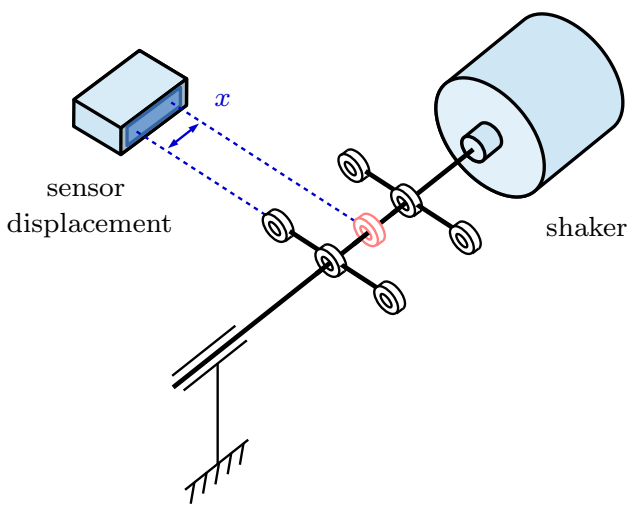

Fig. 7 Experimental set-up for dynamical measurements. The external frame of the MVA (shown in black) is mounted on the shaker. The displacement of the mobile magnet (shown in red) is measured relatively to the displacement of the external frame.

As shown in Fig. 7, the external frame of the MVA is excited using a LDS V455 shaker and the displacement $x$ of the magnet 1 is measured with a Keyence LS-7070M optical sensor. The absorber is placed horizontally to avoid the gravity effect. Indeed, the restoring force would be asymmetric in case of a vertical MVA. The force applied on the vibrating magnet is harmonic. For a given experiment, the amplitude of the forcing is fixed and the frequency is varied. Increasing and decreasing values of the forcing frequency are used in a step-by-step experiment, as is usual for nonlinear vibrations where hysteretic behaviours are awaited due to the presence of multiple equilibria for a certain frequency range. Using this experimental set-up, the equation of motion for the vibrating magnet reads

$$
m_{\text {mag }} \ddot{x}+\chi_{v i s c} \dot{x}+g \mu_{\text {fric }} m_{\text {mag }} \operatorname{sgn}(\dot{x})+K_{1} x+K_{3} x^{3}+K_{5} x^{5}=\mathcal{F}_{\text {ext }} \sin (\Omega t),
$$

with $m_{\text {mag }}$ the mass of the vibrating magnet, $\chi_{v i s c}$ a viscous damping coefficient, $\mu_{\text {fric }}$ a dry friction coefficient (and $\operatorname{sgn}($.$) represents the sign function), g$ the gravitational acceleration, $K_{1}, K_{3}, K_{5}$ the stiffness coefficients and $\mathcal{F}_{\text {ext }}$ and $\Omega$ the amplitude and the frequency of the external force respectively. The identification of all the model parameters (namely $m_{m a g}, \mu_{f r i c}, K_{1}, K_{3}, K_{5}$ and $\chi_{v i s c}$ ) is realised as follows. The mass of the vibrating magnet $m_{m a g}$ is directly measured. The dry friction coefficient $\mu_{f r i c}$ is found by comparing experimental measurements and theoretical values. A set of specific measurements have been realised by applying a static force of increasing amplitude on a free magnet placed on the same lubricated plastic rod as those used for the MVA. The measured force threshold initiating the magnet displacement gives approximately $\mu_{\text {fric }} \approx 0.4$ with an accurate repeatability. This value is exactly within the generic range of static frictional coefficient between lubricated Plexiglas and steel $(0.4-0.5)$ found in tables. Therefore, the value of 0.4 has been retained for all numerical simulations.

Then, one defines the selected geometry for the MVA in order to use it as an NLTVA, an NES or a bi-stable absorber. These 3 arrangements are given fixing the geometric parameters $r$ and $d$ (with $R$ selected as $r-L$ ). From these parameters, together with the magnetic moments derived from the static measurements, the theoretical model using the multipole expansion of section 2 provides the stiffnesses $K_{1}, K_{3}$ and $K_{5}$. The values of these parameters are given in Table 4 for the 3 configurations .

The last parameter to evaluate is the viscous damping coefficient $\chi_{v i s c}$. This coefficient includes all loss mechanisms which are not detailed in the model. For example, these losses could be due to lubricated viscous friction, 


\begin{tabular}{cccc} 
Param. & NLTVA $(\S 4.2 .1)$ & NES $(\S 4.2 .2)$ & Bi-stable absorber $(\S 4.2 .3)$ \\
\hline \hline$r[\mathrm{~mm}]$ & 38 & 47 & 37.5 \\
$d[\mathrm{~mm}]$ & 38 & 24 & 16 \\
\hline$m_{\operatorname{mag}}[\mathrm{g}]$ & 10 & 10 & 6.6 \\
$\chi_{\text {visc }}\left[\mathrm{kg} / \mathrm{s}^{1}\right]$ & 0.09 & 0.044 & 0.079 \\
$\mu_{\text {fric }}[]$ & 0.4 & 0.4 & 0.4 \\
$K_{1}[\mathrm{~N} / \mathrm{m}]$ & 70.49 & -2.11 & -44.22 \\
$K_{3}\left[\mathrm{~N} / \mathrm{m}^{3}\right]$ & $1.3610^{5}$ & $0.7310^{5}$ & $2.5710^{5}$ \\
$K_{5}\left[\mathrm{~N} / \mathrm{m}^{5}\right]$ & $1.2710^{8}$ & $1.2810^{8}$ & $12.9810^{8}$
\end{tabular}

Table 4 Characteristics of the studied configurations.

heat transfer and eddy currents. As the damping behaviour of the MVA in free vibration is dominated by the dry friction for free oscillations, $\chi_{v i s c}$ is difficult to evaluate experimentally by using free decay tests. Therefore, the value of the viscous damping coefficient has been estimated by fitting measured and theoretical frequency response curve. Experimental frequency-response curves are obtained with a step-by-step increase and decrease of the excitation frequency (with a step of $0.5 \mathrm{~Hz}$ ). Numerical solutions are found by continuation, using a pseudo-arclength continuation method implemented in the software AUTO [8]. All the values used to feed the model are given in Table 4. One can observe that slight variations of the viscous damping coefficients are found. They should be due to different physical mechanisms : eddy currents in the fixed magnets are present due to the oscillations of magnet 1 . These damping forces depend on the configuration used so that finding different values of $\chi_{v i s c}$ appears logical. Finally the lubrication on the axis changes slightly from one experiment to another one, once again explaining the observed differences.

\subsection{Results}

\subsubsection{The NLTVA}

First, the MVA is tuned as a TVA with a positive linear stiffness $K_{1}$. The case is termed "NLTVA" as we are also interested in the nonlinear characteristics of the MVA, which have been chosen positive for this case, see the values given in Table 4, second column. Fig. 8 shows the frequency-response curves for five different

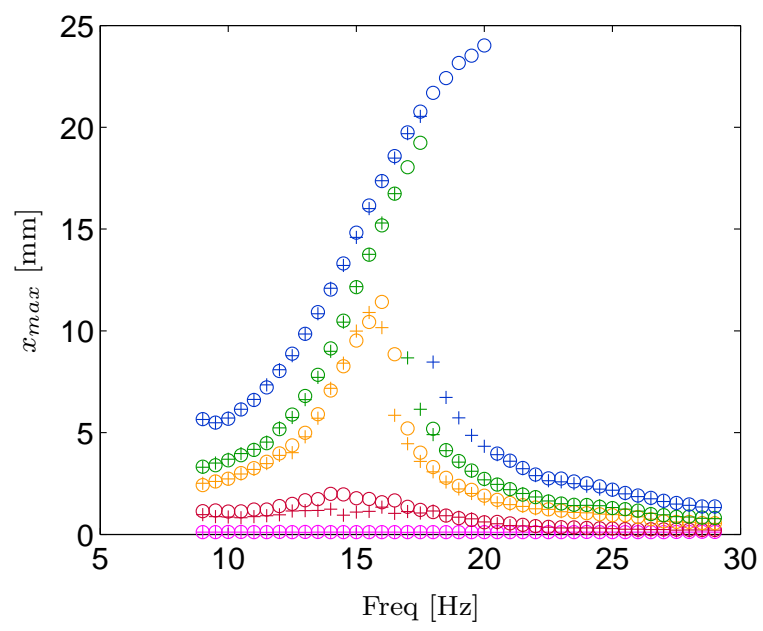

(a)

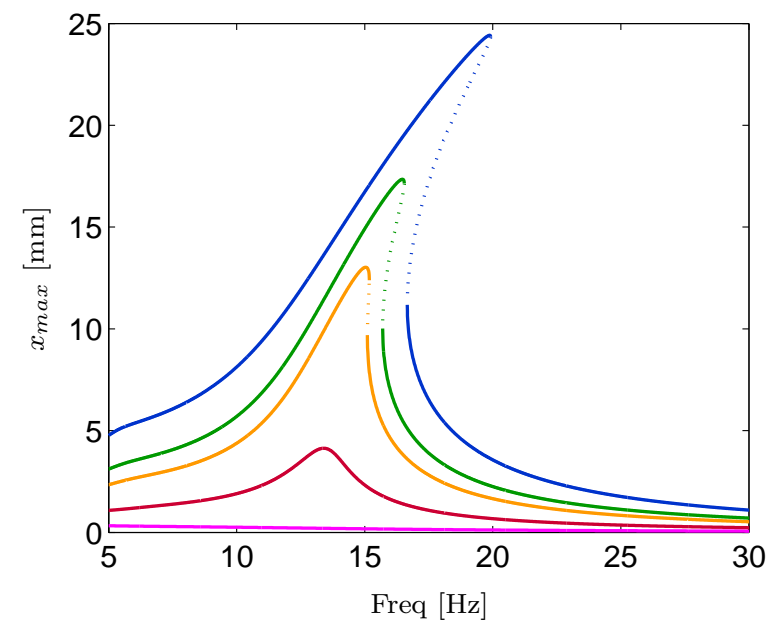

(b)

Fig. 8 MVA tuned as an NLTVA. Maximum amplitude of the displacement of the vibrating magnet for increasing external forces:

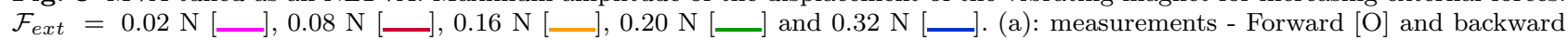
$[+]$ sweeps. (b): simulations. continuous line: stable solutions, dotted lines: unstable solutions.

amplitudes of the forcing, for a frequency range in the vicinity of the eigenfrequency of the MVA oscillator. For each amplitude of the external force, experimental and numerical frequency response curves give very close results. The typical backbone curve due to nonlinear terms appears when the external force is increased. As the $K_{3}$ and $K_{5}$ are both positive, a hardening nonlinearity is observed. In order to illustrate the tuning of the resonance frequency and of the backbone curve, Fig. 9 shows several NLTVA configurations for the same external force $\mathcal{F}_{\text {ext }}=0.32 \mathrm{~N}$. These examples demonstrate that the frequency response of the MVA greatly depends on its geometric configuration. According to Table 5 , the frequency of the peaks is tuned by $K_{1}$ and 


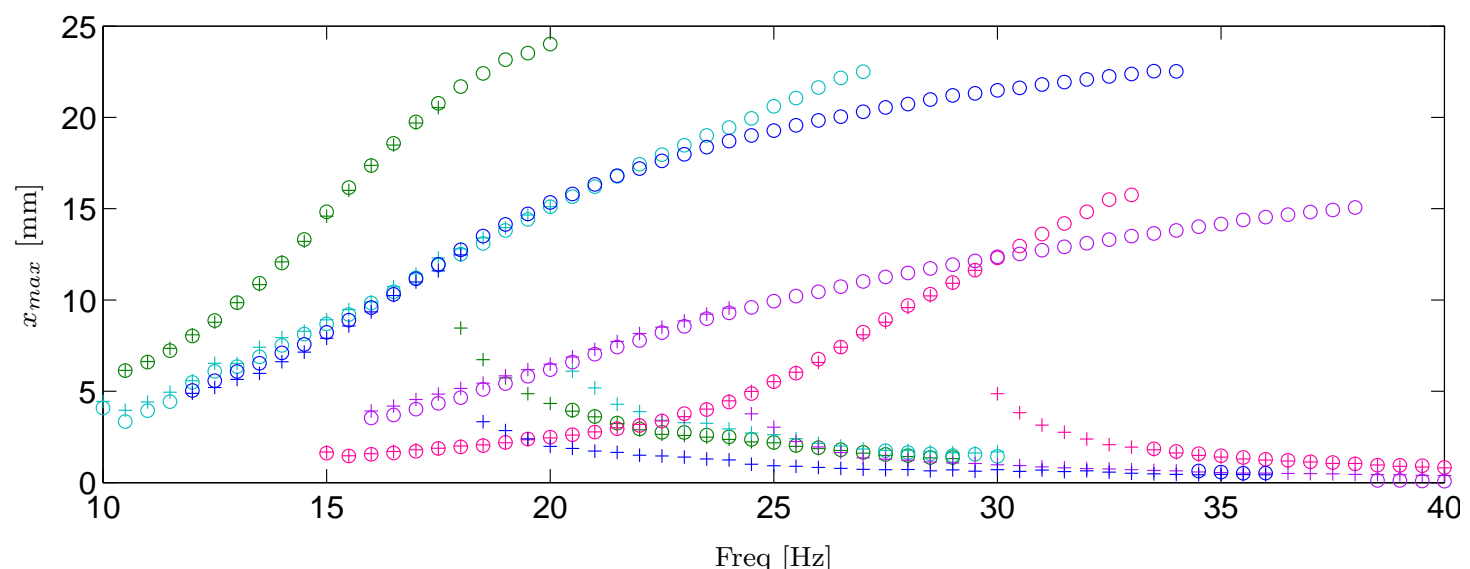

Fig. 9 Examples of experimental NLTVA behaviour for $(r, d)=(38,38) \mathrm{mm}[\mathrm{O}],(36,26) \mathrm{mm}[\mathrm{O}],(30,66) \mathrm{mm}[\mathrm{O}],(30,20) \mathrm{mm}[\mathrm{O}]$ and $(30,27.5) \mathrm{mm}[\mathrm{O}]$. Forward sweeps with circles [O] and backward sweeps with $[+]$.

the nonlinear terms saturate the large amplitudes of the vibrations of the MVA. It must be noticed that the

\begin{tabular}{cccccc} 
Legend & $\mathrm{O}$ & $\mathrm{O}$ & $\mathrm{O}$ & $\mathrm{O}$ & $\mathrm{O}$ \\
\hline$r[\mathrm{~mm}]$ & 38 & 36 & 30 & 30 & 30 \\
$d[\mathrm{~mm}]$ & 38 & 26 & 66 & 20 & 27.5 \\
\hline$K_{1}[\mathrm{~N} / \mathrm{m}]$ & 70.49 & 93.96 & 118.44 & 183.75 & 260.26 \\
$K_{3} 10^{5}\left[\mathrm{~N} / \mathrm{m}^{3}\right]$ & 1.36 & 4.75 & 5.20 & 21.49 & 8.72 \\
$K_{5} 10^{8}\left[\mathrm{~N} / \mathrm{m}^{5}\right]$ & 1.27 & 4.63 & 12.41 & 48.78 & 2.65
\end{tabular}

Table 5 Stiffness coefficients for the NLTVA examples of Fig. 9.

maximum amplitude of the vibrating magnet depends on the geometric configuration. Indeed, this amplitude is obviously limited by $r$. As shown in Fig. 4, largest values of $K_{1}$ are given for small values of $r$. Thus, it seems not possible to have large vibrating amplitude in high frequency. However, this can be overcome by modifying other parameters like magnet dimensions or magnetic characteristics.

\subsubsection{The NES}

The MVA is now tuned as an NES, the characteristics of which are given in the third column of Table 4. Measurements and simulation results are shown in Fig. 10. Due to intrinsic limitations of the shaker used in the experiments, applying a constant external force in low frequency is out of reach, so that the smallest tested excitation frequency is $10 \mathrm{~Hz}$. Below this value, the behaviour of the NES can be studied using the simulation results.

At first sight, the general behaviour of the frequency-response curves share similarities with the case of the NLTVA, the main difference being the vanishing resonance frequency, implying a different behaviour of the solution branches in the low frequency part. However, a main difference is found : the lower branch in high frequencies presents an instability. More precisely, a pitchfork bifurcation point is found numerically for a frequency slightly larger than the saddle-node usual limit point (see Fig. 10(a) where the bifurcation point is indicated with a circle as PF for pitchfork bifurcation). From this bifurcation point, the lower branch is unstable and a couple of stable periodic solutions arise. It has also been found numerically that the basins of attraction of the stable periodic orbits emanating from the pitchfork are very small. Hence, for these parameter values, three solutions coexist in the phase space : a quasi-periodic solution and two stable periodic orbits. The solution that is most likely to be observed being the quasi-periodic one. In fact the only way to observe the periodic solutions emanating from the pitchfork is to continue the solutions slowly from the bifurcation point with increasing excitation frequencies. Only a small portion of the lower branch is stable, in the vicinity of the saddle-node bifurcation point. Thus in an experiment with increasing and decreasing step-by-step harmonic excitation, the following observations are expected:

- when increasing the excitation frequency, the upper branch with large amplitude is followed, until the jump point. At this point, the quasi-periodic regime is expected.

- when decreasing the excitation frequency, the quasi-periodic regime is first observed. Then after the pitchfork bifurcation point and before the jump to the upper branch at the saddle-node point, periodic orbits are observed, corresponding to the stable part of the lower branch. Finally the jump to the upper branch is observed. 


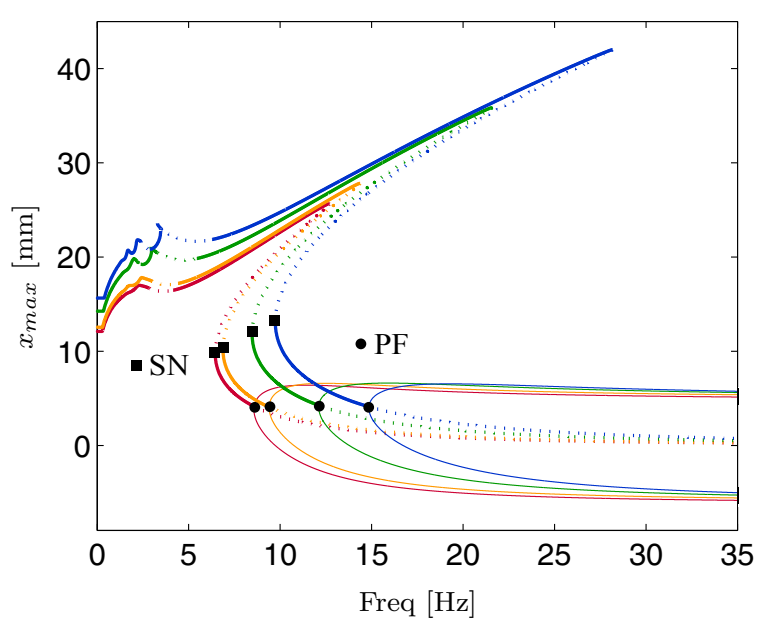

(a)

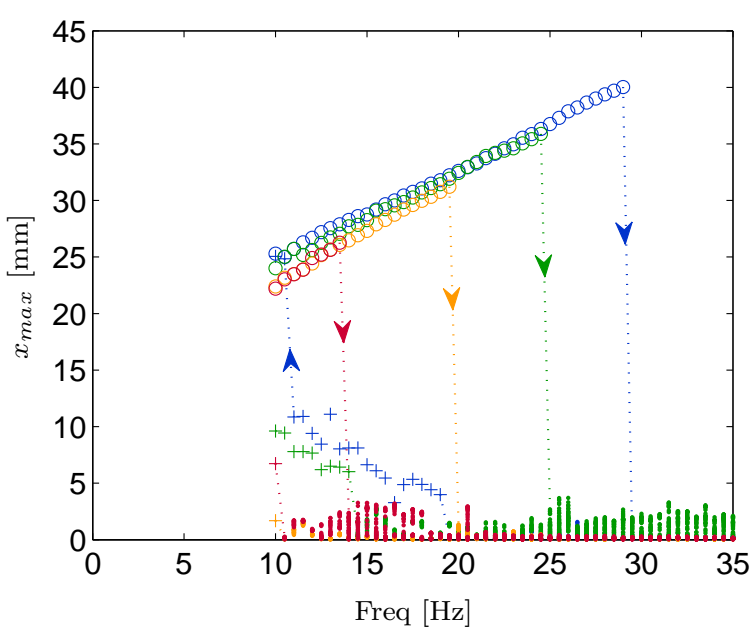

(b)

Fig. 10 MVA tuned as an NES. Maximum amplitude of the displacement of the vibrating magnet for increasing external forces:

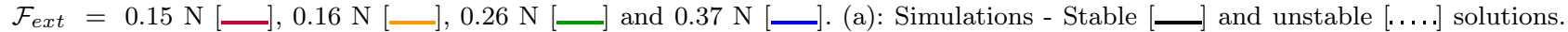
SN: saddle-node bifurcation point. PF: pitchfork bifurcation point. (b): Measurements - Maximum of periodic orbits represented by circles [O] for increasing excitations and crosses [+] for decreasing excitations. When a quasi-periodic regime is observed, a Poincaré section is used to represent the data with dots [.].

Interestingly, the predicted stable and unstable areas are also found in measurements with a very good accuracy as compared to the prediction, see Fig. 10(b). In the tests with increasing excitation frequencies, the upper branch is observed until the jump, then the quasi-periodic solution is observed and represented thanks to a Poincaré section in Fig. 10(b), with a stroboscopic acquisition at the forcing frequency. One can observe that the range of amplitudes explored by the quasi-periodic regime is rather limited, as shown by the extent of the cloud of points from the Poincaré section. This is illustrated in Fig. 11, which represents 10 seconds of the mass displacement, for an excitation frequency of $30 \mathrm{~Hz}$ and an amplitude of $0.26 \mathrm{~N}$. This motion is composed of a fast oscillation with the same frequency than the forcing, modulated by an envelope oscillating at comparatively smaller frequencies. While the amplitude of the fast oscillations are about $1 \mathrm{~mm}$, the modulation amplitude is about $6 \mathrm{~mm}$.

Finally, when decreasing the excitation frequency in Fig. 10(b), the quasi-periodic is at hand. The pitchfork bifurcation point is retrieved experimentally as a small portion of periodic orbits are found before the jump to the upper branch (denoted with crosses in Fig. 10(b)).

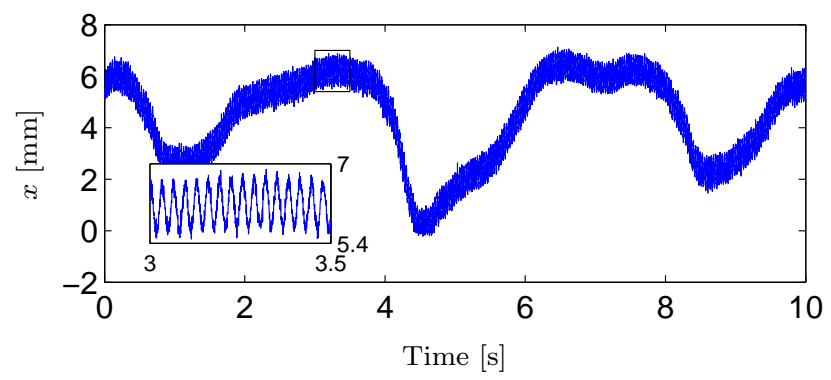

Fig. 11 Time response of the vibrating magnet for the MVA tuned as an NES, for $\Omega=30 \mathrm{~Hz}$ and $\mathcal{F}_{\text {ext }}=0.26 \mathrm{~N}$, for which the quasi-periodic motion is observed. Insert shows a zoomed portion for a better visualisation of the fast oscillation at the forcing frequency.

\subsubsection{The Bi-stable Absorber}

Finally, the MVA is tuned as a bi-stable absorber, the characteristics of which are given in the last column of Table 4. Due to the bi-stability, the behaviour of such a damper is characterised by three distinct regimes depending on the amplitude of the external force. In order to describe these regimes, Fig. 12 and 13 present respectively frequency response curves and the corresponding time evolutions and phase plots of the vibrating mass. 

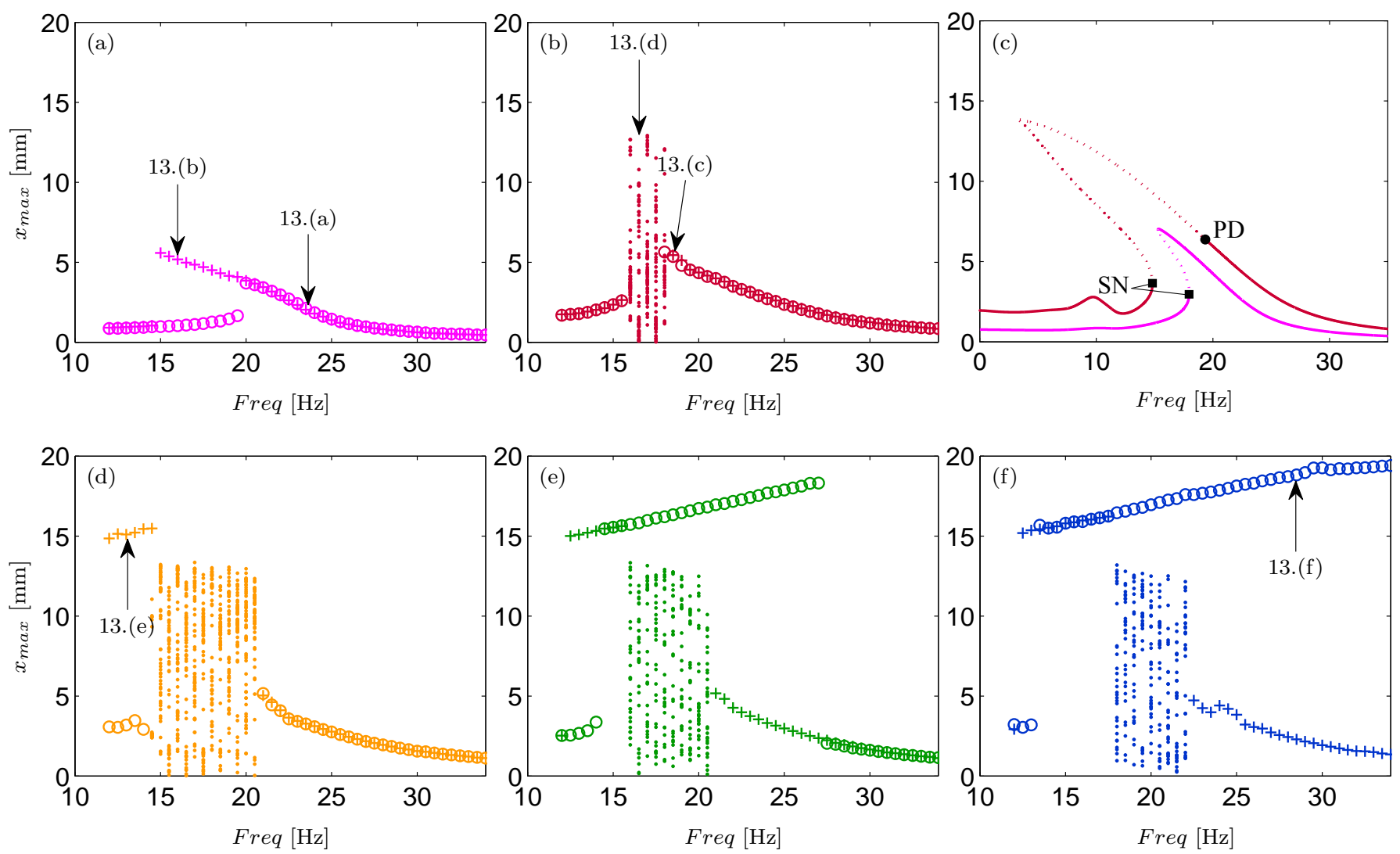

Fig. 12 Maximum amplitude of the displacement of the vibrating magnet configurated as a bi-stable absorber. (a), (b), (d-f): experiments, forward $[\mathrm{O}]$ and backward $[+]$ sweeps and for several external forces. $\left.\mathcal{F}_{\text {ext }}=0.08 \mathrm{~N}[]_{-}\right], 0.16 \mathrm{~N}[-], 0.20 \mathrm{~N}$ [_] $0.23 \mathrm{~N} \mathrm{[ \_}$ ] and $0.24 \mathrm{~N}$ [_] . Dots [.] are the Poincaré sections and arrows indicate the dynamic regimes detailed in Fig. 13. (c): numerical simulation for $\mathcal{F}_{\text {ext }}=0.08 \mathrm{~N}\left[{ }_{-}\right]$, and $0.16 \mathrm{~N}\left[{ }_{-}\right]$, unstable states reported with dotted lines. SN: saddle-node bifurcation, PD : period-doubling.

For small forcing amplitude, the vibrating mass is arbitrarily trapped in one of the two energy wells located at stable equilibria shown in Fig. 5(c). This regime is named intrawell oscillations and the corresponding frequency response is presented in Fig. 12(a). In this regime, a softening type nonlinearity is at hand. Fig. 13(a) shows the time evolution and phase plot of this low energy oscillation. The computation of the central positions of the wells from the model predicts $x_{\text {well }}= \pm 10.5 \mathrm{~mm}$, which is close to the experimental value given in Fig. 13(a) and equal to $\pm 8.9 \mathrm{~mm}$. By increasing the oscillation amplitudes, though still observing them in the intrawell oscillations, one can see that the limit cycle tends to the homoclinic trajectory, so that the measured displacement, indicated with the arrow located on the upper branch and detailed in Fig. 13(b), departs from a sinusoidal motion with increasing amplitudes of the harmonics.

Fig. 12(b) considers the case of a slightly larger external force, showing a more complex behaviour in the frequency range $[15,18] \mathrm{Hz}$. In this region periodic orbits have become unstable in favour of a chaotic motion. The chaotic oscillations are represented by means of Poincaré sections in Fig. 12(b). Interestingly, when decreasing the excitation frequency, a period-doubling is first observed and reported in Fig. 13(c). The chaotic motion, shown in Fig. 13(d), is characterized by aperiodic oscillations between the two wells. Once again the numerical model is able to recover the characteristics of these intrawell frequency-response curves, as shown in Fig. 12(c). In particular, the correct amplitudes are predicted, and the period-doubling bifurcation point is also found in the simulation, leading to the large frequency range at centre of the frequency band where no stable periodic orbits exist anymore.

The external force is increased again in Fig. 12(d) leading to a wider frequency range of unstable oscillations. Moreover, in low frequency the motion jumps on an upper branch during the backward sweep. The amplitude of this new periodic regime, named interwell oscillations and detailed in Fig. 13(e), is large. The magnet now oscillates between the two stable positions with a periodic motion.

As shown in Fig. 12(e) and 12(f), no aperiodic oscillation occurs during the forward sweep for largest external forces. Indeed, the motion jumps on the upper branch in the low frequency range, and the nonlinear coefficients lead to a hardening behaviour. For large amplitudes, the magnet displacement is shown in Fig. 13(f). Finally, the larger the force, the shorter the frequency range of the unstable oscillations and the longer the upper branch 

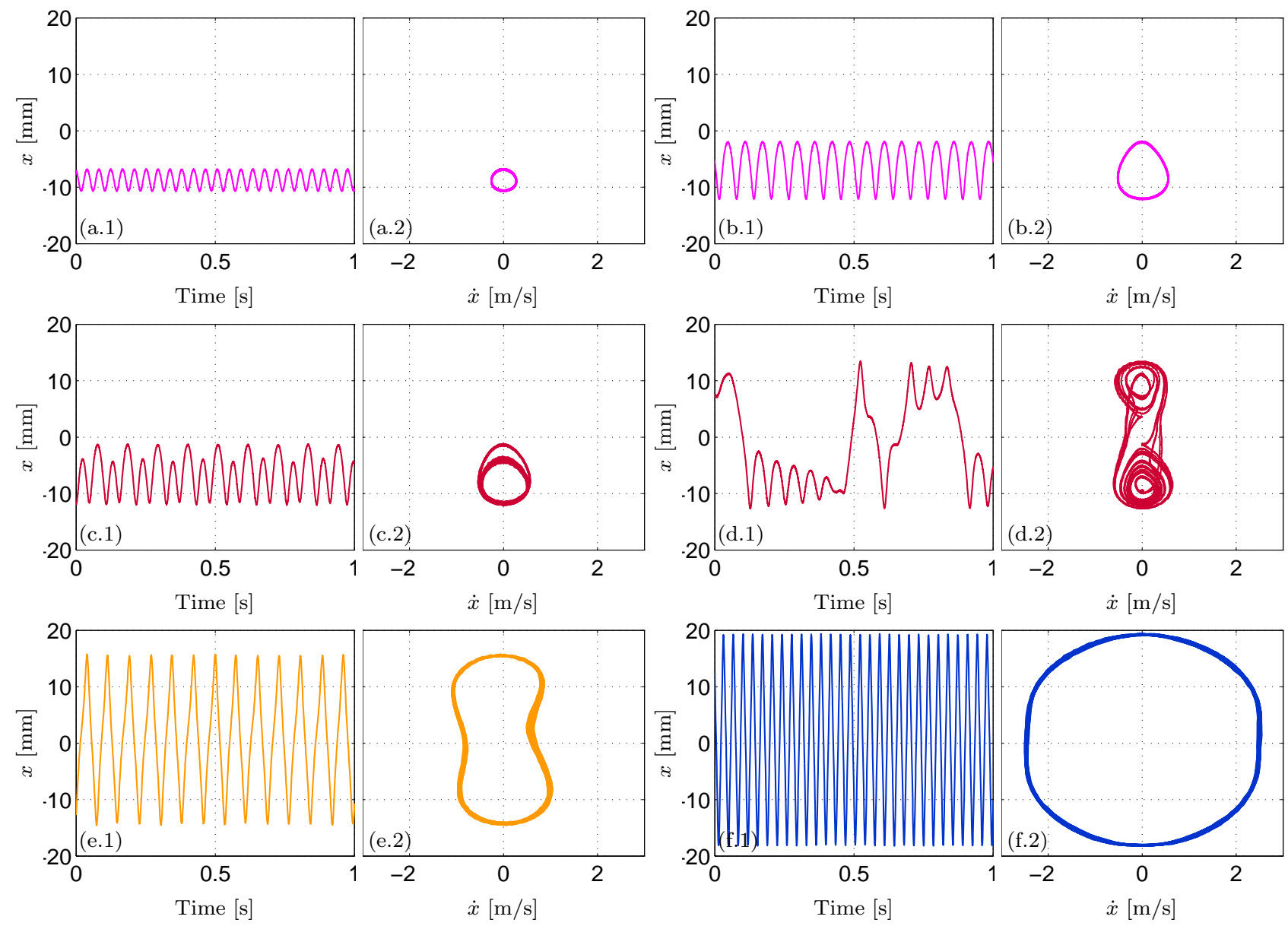

Fig. 13 Experimental time evolutions (1) and phase plots (2) extracted from measurements indicated with arrows in Fig. 12 and illustrating intrawell $(\mathrm{a}, \mathrm{b}, \mathrm{c})$, aperiodic $(\mathrm{d})$ and interwell $(\mathrm{e}, \mathrm{f})$ oscillations. $\mathcal{F}_{\text {ext }}=0.08 \mathrm{~N}[\square], 0.16 \mathrm{~N}[\square], 0.20 \mathrm{~N}[\square]$ and $0.24 \mathrm{~N}$ [ $]$.

in each direction are. It is interesting to notice that the aperiodic vibrations favour the jump on the upper branch during the backward sweep.

\section{Conclusion}

This contribution proposes the design and full characterisation of a passive magnetic vibration absorber (MVA), relying completely on magnetic forces. The mobile mass of the absorber oscillates in a magnetic field created by a set of repulsive and corrective fixed magnets. The linear and nonlinear stiffness coefficients of this original device can be tuned easily, ensuring a great flexible use in different mechanical contexts. This flexibility has been assessed by tuning it either as an NLTVA, an NES or a bi-stable absorber with negative linear stiffness. A model for the absorber has been derived using a multipole expansion of the magnetic field, the validity of which has been verified by means of static measurements. The model predicts the variations of the linear, cubic and quintic stiffness coefficients of the absorber, for each geometric configuration. The dynamical behaviour of the MVA has been also completely characterized in its three main tunings of interest, showing once again the robustness of the model as compared to the measurements. The proposed design appears as an efficient absorber for vibration mitigation which should be easily used in a wide variety of context. Moreover, its versatility should allow one to use the device with different damping properties in a completely passive manner, so that its properties can be changed, depending on the targeted example, or even during the lifetime of a structure. The next steps of this research will consist in testing the MVA as an effective absorber mounted on a real structure. It will also allow us to draw a comparative study of the three families of absorber (NLTVA, NES and bi-stable), in order to test their relative merits and drawbacks on the same example given by a nonlinear structure.

\section{Acknowledgments}

This work was supported by the Direction Générale de l'Armement (DGA) under the grant AMORMAG. 


\section{Appendix A}

This appendix gives the details of the computation of the stiffness coefficients. As the corrective magnets of the MVA presented in Fig. 2 is made up of 3 magnets bonded together, the corresponding coefficients are multiplied by 3 . Moreover, the magnetic moments $m_{c}$ and $m_{r}$ of the corrective and repulsive magnets respectively are different values and are used for the optimisation presented in section 3.2. The stiffness coefficients of Eq. (10) come from the dipolar, quadrupolar, hexapolar and octopolar terms of the magnetic field of the repulsive and corrective magnets and are given by

$$
\begin{aligned}
& K_{1}^{\mathrm{rep}}=K_{1}^{\text {dip,rep }}+K_{1}^{\text {quad,rep }}+K_{1}^{\text {hex,rep }}+K_{1}^{\text {oct }, r e p}, \\
& K_{1}^{\mathrm{cor}}=3\left(K_{1}^{\text {dip,cor }}+K_{1}^{\text {quad,cor }}+K_{1}^{\text {hex }, \text { cor }}+K_{1}^{\text {oct }, c o r}\right), \\
& K_{3}^{\mathrm{rep}}=K_{3}^{\text {dip,rep }}+K_{3}^{q u a d, r e p}+K_{3}^{\text {hex,rep }}+K_{3}^{\text {oct }, r e p} \text {, } \\
& K_{3}^{\text {cor }}=3\left(K_{3}^{\text {dip,cor }}+K_{3}^{q u a d, c o r}+K_{3}^{\text {hex }, \text { cor }}+K_{3}^{\text {oct }, c o r}\right) \text {, } \\
& K_{5}^{\mathrm{rep}}=K_{5}^{\text {dip,rep }}+K_{5}^{\text {quad,rep }}+K_{5}^{\text {hex,rep }}+K_{5}^{\text {oct,rep }} \text {, } \\
& K_{5}^{\text {cor }}=3\left(K_{5}^{\text {dip,cor }}+K_{5}^{\text {quad,cor }}+K_{5}^{\text {hex,cor }}+K_{5}^{\text {oct }, \text { cor }}\right) \text {, }
\end{aligned}
$$

with

$$
\begin{aligned}
& K_{1}^{\text {dip,rep }}=48 c_{\text {dip }} m_{r} \frac{1}{r^{5}}, \quad K_{3}^{\text {dip,rep }}=240 c_{\text {dip }} m_{r} \frac{1}{r^{7}}, \quad K_{5}^{\text {dip,rep }}=672 c_{\text {dip }} m_{r} \frac{1}{r^{9}}, \\
& K_{1}^{\text {quad }, r e p}=480 c_{\text {quad }} m_{r} \frac{1}{r^{7}}, \quad K_{3}^{\text {quad,rep }}=4480 c_{\text {quad }} m_{r} \frac{1}{r^{9}}, \quad K_{5}^{\text {quad,rep }}=20160 c_{\text {quad }} m_{r} \frac{1}{r^{11}}, \\
& K_{1}^{\text {hex }, \text { rep }}=1792 c_{\text {hex }} m_{r} \frac{1}{r^{9}}, \quad K_{3}^{\text {hex }, \text { rep }}=26880 c_{\text {hex }} m_{r} \frac{1}{r^{11}}, \quad K_{5}^{\text {hex,rep }}=177408 c_{\text {hex }} m_{r} \frac{1}{r^{13}}, \\
& K_{1}^{o c t, r e p}=23040 c_{o c t} m_{r} \frac{1}{r^{11}}, \quad K_{3}^{\text {oct }, r e p}=506880 c_{o c t} m_{r} \frac{1}{r^{13}}, \quad K_{5}^{\text {oct }, r e p}=4612608 c_{o c t} m_{r} \frac{1}{r^{15}},
\end{aligned}
$$

and

$$
\begin{aligned}
& K_{1}^{d i p, c o r}=12 c_{d i p} m_{c} \frac{\left(8 R^{4}-24 d^{2} R^{2}+3 d^{4}\right)}{\left(R^{2}+d^{2}\right)^{9 / 2}}, \\
& K_{1}^{\text {quad,cor }}=60 c_{\text {quad }} m_{c} \frac{\left(16 R^{6}-120 R^{4} d^{2}+90 R^{2} d^{4}-5 d^{6}\right)}{\left(R^{2}+d^{2}\right)^{13 / 2}}, \\
& K_{1}^{\text {hex }, \text { cor }}=28 c_{\text {hex }} m_{c} \frac{\left(128 R^{8}-1792 R^{6} d^{2}+3360 R^{4} d^{4}-1120 R^{2} d^{6}+35 d^{8}\right)}{\left(R^{2}+d^{2}\right)^{17 / 2}}, \\
& K_{1}^{\text {oct }, \text { cor }}=180 c_{\text {oct }} m_{c} \frac{\left(256 R^{10}-5760 R^{8} d^{2}+20160 R^{6} d^{4}-16800 R^{4} d^{6}+3150 R^{2} d^{8}-63 d^{10}\right)}{\left(R^{2}+d^{2}\right)^{21 / 2}}, \\
& K_{3}^{\text {dip,cor }}=30 c_{\text {dip }} m_{c} \frac{\left(16 R^{6}-120 d^{2} R^{4}+90 d^{4} R^{2}-5 d^{6}\right)}{\left(R^{2}+d^{2}\right)^{13 / 2}}, \\
& K_{3}^{\text {quad,cor }}=70 c_{\text {quad }} m_{c} \frac{\left(128 R^{8}-1792 R^{6} d^{2}+3360 R^{4} d^{4}-1120 R^{2} d^{6}+35 d^{8}\right)}{\left(R^{2}+d^{2}\right)^{17 / 2}}, \\
& K_{3}^{\text {hex }, \text { cor }}=210 c_{\text {hex }} m_{c} \frac{\left(256 R^{10}-5760 R^{8} d^{2}+20160 R^{6} d^{4}-16800 R^{4} d^{6}+3150 R^{2} d^{8}-63 d^{10}\right)}{\left(R^{2}+d^{2}\right)^{21 / 2}}, \\
& K_{3}^{\text {oct }, \text { cor }}=990 c_{\text {oct }} m_{c} \frac{\left(1024 R^{12}-33792 R^{10} d^{2}+190080 R^{8} d^{4}-295680 R^{6} d^{6}+138600 R^{4} d^{8}-16632 R^{2} d^{10}+231 d^{12}\right)}{\left(R^{2}+d^{2}\right)^{25 / 2}}, \\
& K_{5}^{d i p, c o r}=\frac{21}{2} c_{d i p} m_{c} \frac{\left(128 R^{8}-1792 d^{2} R^{6}+3360 d^{4} R^{4}-1120 d^{6} R^{2}+35 d^{8}\right)}{\left(R^{2}+d^{2}\right)^{17 / 2}}, \\
& K_{5}^{\text {quad }, \text { cor }}=\frac{315}{2} c_{\text {quad }} m_{c} \frac{\left(256 R^{10}-5760 R^{8} d^{2}+20160 R^{6} d^{4}-16800 R^{4} d^{6}+3150 R^{2} d^{8}-63 d^{10}\right)}{\left(R^{2}+d^{2}\right)^{21 / 2}} \text {, } \\
& K_{5}^{\text {hex }, \text { cor }}=\frac{693}{2} c_{\text {hex }} m_{c} \\
& \frac{\left(1024 R^{12}-33792 R^{10} d^{2}+190080 R^{8} d^{4}-295680 R^{6} d^{6}+138600 R^{4} d^{8}-16632 R^{2} d^{10}+231 d^{12}\right)}{\left(R^{2}+d^{2}\right)^{25 / 2}}, \\
& K_{5}^{\text {oct }, \text { cor }}=\frac{9009}{2} c_{\text {oct }} m_{c} \\
& \underline{\left(2048 R^{14}-93184 R^{12} d^{2}+768768 R^{10} d^{4}-1921920 R^{8} d^{6}+1681680 R^{6} d^{8}-504504 R^{4} d^{10}+42042 R^{2} d^{12}-429 d^{14}\right)} \\
& \left(R^{2}+d^{2}\right)^{29 / 2}
\end{aligned}
$$


where

$$
\begin{aligned}
& c_{\text {dip }}=\frac{\mu_{0} m_{1}}{4 \pi} \\
& c_{\text {quad }}=\frac{\mu_{0} m_{1}}{4 \pi}\left(\frac{L}{2}\right)^{2} \frac{3\left(\beta_{\text {int }}^{2}-\beta_{\text {ext }}^{2}\right)}{8}, \\
& c_{\text {hex }}=\frac{\mu_{0} m_{1}}{4 \pi}\left(\frac{L}{2}\right)^{4} \frac{15\left(\beta_{\text {ext }}^{4}-\beta_{\text {int }}^{4}\right)+60\left(\beta_{\text {int }}^{2}-\beta_{\text {ext }}^{2}\right)}{64}, \\
& c_{\text {oct }}=\frac{\mu_{0} m_{1}}{4 \pi}\left(\frac{L}{2}\right)^{6} \frac{35\left(\beta_{\text {int }}^{6}-\beta_{\text {ext }}^{6}\right)+280\left(\beta_{\text {ext }}^{4}-\beta_{\text {int }}^{4}\right)+336\left(\beta_{\text {int }}^{2}-\beta_{\text {ext }}^{2}\right)}{1024},
\end{aligned}
$$

and $\beta_{\text {int }}=\frac{D_{i n t}}{L}$ and $\beta_{\text {ext }}=\frac{D_{e x t}}{L}$. 


\section{References}

1. Al-Shudeifat, M.: Asymmetric magnet-based nonlinear energy sink. J. Comput. Nonlinear Dynam 10(1), 014,502 (2014)

2. AL-Shudeifat, M.A., Wierschem, N., Quinn, D.D., Vakakis, A.F., Bergman, L.A., Jr., B.F.S.: Numerical and experimental investigation of a highly effective single-sided vibro-impact non-linear energy sink for shock mitigation. International Journal of Non-Linear Mechanics 52, 96-109 (2013)

3. Asami, T., Nishihara, O., Baz, A.: Analytical solutions to $\mathrm{H}_{\infty}$ and $\mathrm{H}_{2}$ optimization of dynamic vibration absorbers attached to damped linear systems. Journal of Vibration and Acoustics 124, 284-295 (2002)

4. Bourquin, F., Caruso, G., Peigney, M., Siegert, D.: Magnetically tuned mass damper for optimal vibration damping of large structures. Smart Materials and Structures 23, 13pp (2014)

5. Carrella, A., Brennan, M., Kovacic, I., Waters, T.: On the force transmissibility of a vibration isolator with quasi-zero-stiffness. Journal of Sound and Vibration 322(4-5), 707-717 (2009)

6. Carrella, A., Brennan, M., Waters, T.: Static analysis of a passive vibration isolator with quasi-zero-stiffness characteristic. Journal of Sound and Vibration 301(3-5), 678-689 (2007)

7. Carrella, A., Brennan, M., Waters, T., Shin, K.: On the design of a high-static-low-dynamic stiffness isolator using linear mechanical springs and magnets. Journal of Sound and Vibration 315(3), 712-720 (2008)

8. Doedel, E.J., Paffenroth, R., Champneys, A.R., Fairgrieve, T.F., Kuznetsov, Y.A., Oldeman, B.E., Sandstede, B., Wang, X.: Auto 2000 : Continuation and bifurcation software for ordinary differential equations. Technical report, Concordia University, 2002

9. Feudo, S.L., Allani, A., Cumunel, G., Argoul, P., Bruno, D.: Experimental tuned mass damper based on eddy currents damping effect and adjustable stiffness. In: CSMA 2015, $12^{e}$ colloque national en calcul des structures. Giens (2015)

10. Frahm, H.: Device for damping vibrations of bodies. Tech. rep., US No. Patent 989958 (1909)

11. Gendelman, O., Manevitch, L., Vakakis, A., Closkey, R.M.: Energy pumping in nonlinear mechanical oscillators, I: dynamics of the underlying hamiltonian systems. Journal of Applied Mechanics 68(1), 34-41 (2001)

12. Gourc, E., Michon, G., Séguy, S., Berlioz, A.: Experimental investigation and design optimization of targeted energy transfer under periodic forcing. Journal of Vibration and Acoustics 136(2), 021,021 (2014)

13. Gourc, E., Michon, G., Séguy, S., Berlioz, A.: Targeted energy transfer under harmonic forcing with a vibro-impact nonlinear energy sink: Analytical and experimental developments. Journal of Vibration and Acoustics 137(3), 031,008 (2015)

14. Gourdon, E., Alexander, N., Taylor, C., Lamarque, C., Pernot, S.: Nonlinear energy pumping under transient forcing with strongly nonlinear coupling: Theoretical and experimental results. Journal of Sound and Vibration 300(3-5), 522-551 (2007)

15. Grappasonni, C., Habib, G., Detroux, T., FengWen, W., Kerschen, G., Jensen, J.: Practical design of a nonlinear tuned vibration absorber. In: Proceedings of the ISMA 2014 conference (2014)

16. Grappasonni, C., Habib, G., Detroux, T., Kerschen, G.: Experimental demonstration of a 3d-printed nonlinear tuned vibration absorber. In: Proceedings of the International Modal Analysis Conference (IMAC) XXXIII. Orlando (2015)

17. Habib, G., Detroux, T., Viguié, R., Kerschen, G.: Nonlinear generalization of den Hartog's equal-peak method. Mechanical Systems and Signal Processing 52-53, 17-28 (2015)

18. Habib, G., Kerschen, G.: Suppression of limit cycle oscillations using the nonlinear tuned vibration absorber. Proceedings of the Royal Society of London A $\mathbf{4 7 1}(2176)$ (2015)

19. Haddow, A.G., Shaw, S.W.: Centrifugal pendulum vibration absorbers: An experimental and theoretical investigation. Nonlinear Dynamics 34(3-4), 293-307 (2003)

20. Hartog, J.D.: Mechanical Vibrations. McGraw-Hill, New-York (1934)

21. Kojima, H., Nagaya, K.: Forced vibrations of a circular plate with a nonlinear dynamic vibration absorber. Bulletin of the JSME 28(136), 309-314 (1985)

22. Kojima, H., Saito, H.: Forced vibrations of a beam with a non-linear dynamic vibration absorber. Journal of Sound and Vibration 88(4), 559-568 (1983)

23. Kovacic, I., Brennan, M.J., Waters, T.P.: A study of a nonlinear vibration isolator with a quasi-zero stiffness characteristic. Journal of Sound and Vibration 315(3), 700-711 (2008)

24. Krenk, S., Hogsberg, J.: Tuned mass absorber on a flexible structure. Journal of Sound and Vibration 333, 1577-1595 (2014)

25. Lamarque, C.H., Gendelman, O.V., Ture Savadkoohi, A., Etcheverria, E.: Targeted energy transfer in mechanical systems by means of non-smooth nonlinear energy sink. Acta Mechanica 221(1-2), 175-200 (2011)

26. Lee, Y.S., Kerschen, G., McFarland, D.M., Hill, W.J., Nichkawde, C., Strganac, T.W., Bergman, L.A., Vakakis, A.F.: Suppressing aeroelastic instability using broadband passive targeted energy transfers, part II: Experiments. AIAA Journal 45(10), 2391-2400 (2007)

27. Lee, Y.S., Vakakis, A.F., Bergman, L.A., McFarland, D.M., Kerschen, G.: Suppressing aeroelastic instability using broadband passive targeted energy transfers, part I: Theory. AIAA Journal 45(3), 693-711 (2007)

28. Liu, X., Huang, X., Hua, H.: On the characteristics of a quasi-zero stiffness isolator using Euler buckled beam as negative stiffness corrector. Journal of Sound and Vibration 332(14), 3359-3376 (2013)

29. Manevitch, L.I., Sigalov, G., Romeo, F., Bergman, L.A., Vakakis, A.F.: Dynamics of a linear oscillator coupled to a bistable light attachment: Analytical study. J. Appl. Mech. 81(4), 041,011 (2013)

30. Mann, B., Owens, B.: Investigations of a nonlinear energy harvester with bistable potential well. Journal of Sound and Vibration 329(9), 1215-1226 (2010)

31. McFarland, D.M., Bergman, L.A., Vakakis, A.F.: Experimental study of non-linear energy pumping occurring at a single fast frequency. International Journal of Nonlinear Mechanics 40, 891-899 (2005)

32. Mehmood, A., Nayfeh, A.H., Hajj, M.R.: Effects of a non-linear energy sink (NES) on vortex-induced vibrations of a circular cylinder. Nonlinear Dynamics 77, 667-680 (2014)

33. Ormondroyd, J., Den Hartog, J.: The theory of the dynamic vibration absorber. Transactions of ASME 50, 9-22 (1928)

34. Pellicano, F., Amabili, M., Païdoussis, M.P.: Effect of the geometry on the non-linear vibration of circular cylindrical shells. International Journal of Non-linear Mechanics 37, 1181-1198 (2002)

35. Petruska, A., Abbott, J.: Optimal permanent-magnet geometries for dipole field approximation. IEEE transactions on magnetics 49(2), 811-819 (2013)

36. Robertson, W.S., Kidner, M., Cazzolato, B.S., Zander, A.C.: Theoretical design parameters for a quasi-zero stiffness magnetic spring for vibration isolation. Journal of Sound and Vibration 326(1-2), 88-103 (2009)

37. Romeo, F., Sigalov, G., Bergman, L.A., Vakakis, A.F.: Dynamics of a linear oscillator coupled to a bistable light attachment: Numerical study. J. Comput. Nonlinear Dynam. 10(1), 011,007 (2014)

38. Sodano, H.A., Bae, J., Inman, D.J., Belvin, W.K.: Concept and model of eddy current damper for vibration suppression of a beam. Journal of Sound and Vibration 288, 1177-1196 (2005) 
39. Touzé, C., Thomas, O.: Non-linear behaviour of free-edge shallow spherical shells: effect of the geometry. International Journal of Non-linear Mechanics 41(5), 678-692 (2006)

40. Tsai, H., Lin, G.: Optimum tuned mass dampers for minimizing steady-state response of support excited and damped system. Earthquake Engineering and Structural Dynamics 11, 846-862 (1993)

41. Vakakis, A., Gendelman, O.: Energy pumping in nonlinear mechanical oscillators, II: resonance capture. Journal of Applied Mechanics 68(1), 42-48 (2001)

42. Vakakis, A., Gendelman, O., Bergman, L., McFarland, D., Kerschen, G., Lee, Y.: Nonlinear targeted energy transfer in mechanical and structural systems I. Springer, New-York (2008)

43. Vakakis, A., Gendelman, O., Bergman, L., McFarland, D., Kerschen, G., Lee, Y.: Nonlinear targeted energy transfer in mechanical and structural systems II. Springer, New-York (2008)

44. Vakakis, A., Manevitch, L., Gendelman, O., Bergman, L.: Dynamics of linear discrete systems connected to local, essentially non-linear attachments. Journal of Sound and Vibration 264, 559-577 (2003)

45. Viguié, R., Kerschen, G.: Nonlinear vibration absorber coupled to a nonlinear primary system: A tuning methodology. Journal of Sound and Vibration 326(3-5), 780-793 (2009)

46. Xu, D., Yu, Q., Zhou, J., Bishop, S.: Theoretical and experimental analyses of a nonlinear magnetic vibration isolator with quasi-zero-stiffness characteristic. Journal of Sound and Vibration 332(14), 3377-3389 (2013)

47. Yamakawa, I., Takeda, S., Kojima, H.: Behavior of a new type dynamic vibration absorber. Bulletin of the JSME 20(146), 947-954 (1977)

48. Zilletti, M., Elliott, S.J., Rustighi, E.: Optimisation of dynamic vibration absorbers to minimise kinetic energy and maximise internal power dissipation. Journal of Sound and Vibration 331, 4093-4100 (2012) 